\title{
High spatial resolution mid-infrared spectroscopy of the starburst galaxies NGC 3256, II Zw 40 and Henize 2-10^
}

\author{
N. L. Martín-Hernández ${ }^{1}$, D. Schaerer ${ }^{2,3}$, E. Peeters ${ }^{4}$, A. G. G. M. Tielens ${ }^{5}$, and M. Sauvage ${ }^{6}$ \\ 1 Instituto de Astrofísica de Canarias, Vía Lactea, 38200 La Laguna, Tenerife, Spain \\ e-mail: leticia@iac.es \\ 2 Observatoire de Genève, 51 Chemin des Maillettes, 1290 Sauverny, Switzerland \\ 3 Laboratoire Astrophysique de Toulouse-Tarbes (UMR 5572), Observatoire Midi-Pyrénées, 14 Avenue E. Belin, \\ 31400 Toulouse, France \\ 4 NASA Ames Research Center, MS 245-6, Moffett Field, CA 94035, USA \\ 5 SRON National Institute for Space Research and Kapteyn Institute, PO Box 800, 9700 AV Groningen, The Netherlands \\ ${ }^{6}$ CEA/DSM/DAPNIA/SAp, CE Saclay, 91191 Gif-sur-Yvette Cedex, France
}

Received 4 October 2005 / Accepted 26 April 2006

\begin{abstract}
Aims. In order to show the importance of high spatial resolution observations of extra-galactic sources when compared to observations obtained with larger apertures such as ISO, we present $N$-band spectra $(8-13 \mu \mathrm{m})$ of some locations in three starburst galaxies. In particular, we show the two galactic nuclei of the spiral galaxy NGC 3256, the compact IR supernebula in the dwarf galaxy II Zw 40 and the two brightest IR knots in the central starburst of the WR galaxy He 2-10.

Methods. The spectra were obtained with TIMMI2 on the ESO $3.6 \mathrm{~m}$ telescope. An inventory of the spectra in terms of atomic fine-structure lines and molecular bands is presented.

Results. We show the value of these high spatial resolution data in constraining properties such as the extinction in the mid-IR, metallicity or stellar content (age, IMF, etc.). We have constrained the stellar content of the IR compact knot in II Zw 40 by using the mid-IR fine-structure lines and setting restrictions on the nebular geometry. We have constructed a new mid-/far-IR diagnostic diagram based on the $11.2 \mu \mathrm{m}$ PAH and continuum, accessible to ground-based observations. We find that extra-galactic nuclei and star clusters observed at high spatial resolution (as is the case of the TIMMI2 observations) are closer in PAH/far-IR to compact $\mathrm{H}$ II regions, while galaxies observed by large apertures such as ISO are closer to exposed PDRs such as Orion. This is likely due to the aperture difference. We find a dependence between the presence of PAHs and the hardness of the radiation field as measured by the $[\mathrm{S} \mathrm{IV}] /[\mathrm{Ne} \mathrm{II}]$ ratio that may be explained by the PAH-dust competition for FUV photons or the relative contribution of the different phases of the interstellar medium.
\end{abstract}

Key words. ISM: lines and bands - dust, extinction - H II regions - galaxies: starburst - infrared: galaxies

\section{Introduction}

Mid-infrared (MIR) observations have proved to be of great value at providing spectral diagnostics to quantify massive star formation and to distinguish between stellar (starburst) and other (AGN) activity (e.g. Genzel \& Cesarsky 2000). However, most of these diagnostics are based on "integrated spectra" which have been obtained through large apertures e.g. with the Infrared Space Observatory, ISO, with an aperture larger than 14" for ISO/SWS (cf. Schaerer \& Stasińska 1999; Thornley et al. 2000; Förster Schreiber et al. 2001; Rigby \& Rieke 2004). Therefore, these measurements are likely to consist of contributions from numerous "knots", the diffuse ISM, etc, rendering their interpretation difficult. Furthermore, some diagnostics (e.g. relating PAH and continuum emission, high and low excitation fine-structure lines) may originate from different spatial regions and in consequence, it is not clear whether, and to what extent, such spatially integrated or "global" spectra can be used for various diagnostic purposes.

^ Based on observations obtained at the European Southern Observatory, La Silla, Chile (ID 70.B-0583).
It has already been demonstrated how the spatial scale of observations greatly determine the MIR appearance of galaxies (e.g. Martín-Hernández et al. 2005; Siebenmorgen et al. 2004). This is the case, for instance, of the starburst galaxy NGC 5253, where most of the high-excitation fine-structure line fluxes measured within the ISO aperture (e.g. [S IV]) are emitted by a single knot of $\sim 0$ ' 1 , while other sources within the aperture mainly contribute to the low-excitation line fluxes. Only $\sim 20 \%$ of the [Ne II] emission comes from the otherwise dominant compact knot (Martín-Hernández et al. 2005). This has important implications for the interpretation of line fluxes in terms of properties of the stellar content such as age, IMF, etc.

We have recently started gathering ground-based high spatial resolution MIR observations of several nearby starbursts using TIMMI2 on the $3.6 \mathrm{~m}$ ESO telescope, which provides a slit width of 1"2. Our pilot work on the well-studied NGC 5253 (Martín-Hernández et al. 2005) demonstrated the value of this type of data in constraining properties such as the extinction in the MIR, metallicity or stellar content (age, IMF, etc.). Here we present observations of some locations in three young starburst galaxies. Two of these, the dwarf galaxy II Zw 40 and the 
WR galaxy He 2-10 are, together with NGC 5253, well-known young starburst galaxies that possess embedded and compact knots likely representing the earliest evolutionary stages of super star clusters (SSCs) or proto-globular clusters (e.g. Turner et al. 1998; Kobulnicky \& Johnson 1999; Beck et al. 2002; Vacca et al. 2002; Johnson \& Kobulnicky 2003). MIR images of these types of galaxies (e.g. Gorjian et al. 2001; Beck et al. 2001) confirm the cluster hypothesis and indicate that a large fraction ( $\gtrsim 30-70 \%)$ of the total IR luminosity may originate from these compact knots. The third starburst galaxy we present here is NGC 3256, a spiral galaxy which is the brightest IR source in the nearby universe. MIR images (Böker et al. 1997) reveal that NGC 3256 has two distinct nuclei aligned in the north-south direction and separated by $5^{\prime \prime}$. The southern nucleus has no optical counterpart. Hence, our observations include the two galactic nuclei of NGC 3256, the bright and compact infrared supernebula in II Zw 40 and the two brightest infrared sources in the central starburst of $\mathrm{He} 2-10$ (named A and $\mathrm{C}$ following the nomenclature of Beck et al. 2001).

The paper is structured as follows. Our observations are described in Sect. 2. Immediate results from our TIMMI2 spectra are shown in Sect. 3. The discussion is presented in Sect. 4. The main conclusions of the paper are summarised in Sect. 5.

\section{Observations and data reduction}

Our new infrared data on NGC 3256, II Zw 40 and He 2-10 were obtained as part of a program to observe young starburst galaxies with the Thermal Infrared MultiMode Instrument (TIMMI2) on the ESO 3.6 m telescope (La Silla Observatory, Chile).

The $N$-band spectra of the nuclear regions of these three starburst galaxies were obtained on 2003 March 20-26. We used the $10 \mu \mathrm{m}$ low-resolution grism which ranges from 7.5 to $13.9 \mu \mathrm{m}$ and has a spectral resolving power $\lambda / \Delta \lambda \sim 160$. The slit used was $11^{\prime \prime} .2 \times 70^{\prime \prime}$, with a pixel scale of 0.45 . At the time of the observations, the slit could only be oriented in the northsouth direction. It was positioned across the bright northern nucleus in the case of NGC 3256 (including as well the southern nucleus), across the bright infrared compact core in the case of II Zw 40 and across the two brightest MIR components in the case of He 2-10, A and C (we refer to Sects. 3.1.1, 3.2.1 and 3.3.1 for a detailed description of the objects). In order to correct for background emission from the sky, the observations were performed using a standard chopping/nodding technique along the slit in the north-south direction (where the object is observed at two different positions on the slit) with an amplitude of $20^{\prime \prime}$ in the case of NGC 3256 and $10^{\prime \prime}$ in the cases of II Zw 40 and $\mathrm{He} 2-10$. Adopted calibration stars were HD 90957 for NGC 3256, HD 37160 for II Zw 40 and HD 75691 and HD 73603 for He 2-10. They were observed right before and after the targets and served as both telluric and flux standard stars. They were used as well for Point Spread Function (PSF) determination. The synthetic calibrated spectra for these standard stars are given by Cohen et al. (1999).

The data processing included the removal of bad frames and the co-addition of all chopping and nodding pairs. This left us with one single image with one positive and two negative longslit spectra. These were combined with a simple shift-and-add procedure which slightly increased the signal-to-noise. For each individual observation, the spectra of the target and standard star were extracted using the optimal extraction procedure developed by Horne (1986), ideal for unresolved or compact sources. This procedure applies non-uniform pixel weights in the extraction sum in order to reduce the statistical noise in the extracted spectrum to a minimum while preserving its photometric accuracy. The calibration of the spectroscopic data included 1) the removal of the telluric features, which was done by dividing by the spectrum of the standard star; 2) the removal of the spectral features of the standard star; and 3) the absolute flux calibration. These last two steps were achieved by multiplying by the synthetic spectrum of the standard star. We propagated the uncertainty for each pixel, dominated by variations of the sky transparency, along each step of the processing.

Wavelength calibration is straightforward since a table with the pixel-to-wavelength correspondence is provided on the TIMMI2 webpage ${ }^{1}$.

We obtained two spectra of NGC 3256, three of II Zw 40, two of He 2-10 A and three of the weaker He 2-10 C. Each spectrum was obtained after a total exposure time (on source) of $\sim 32 \mathrm{~min}$. The spectra of each source were combined to obtain one final spectrum.

We compared the full-width-at-half-maximum ( $F W H M$ ) measured along the spatial direction for each object with the MIR seeing (derived from the spatial FWHM of the calibration star) in order to account for possible slit losses.

The $F W H M$ along the spatial direction measured for the southern nucleus (S) of NGC 3256 is $1{ }^{\prime \prime} 0 \pm 0.2$, which is of the order of the MIR seeing (FWHM 0.'95-1'.11) and smaller than the slit width (1".2). Hence, slit losses towards NGC $3256 \mathrm{~S}$ are negligible. However, the northern nucleus (NGC $3256 \mathrm{~N}$ ) is slightly resolved with a $F W H M$ along the spatial direction of $11^{\prime \prime} 55 \pm 0$.'05. Assuming that the source is Gaussian and that the slit is perfectly centred on the source peak, this implies that the slit might be registering about $68 \%$ of the total emission of the $\mathrm{N}$ nucleus.

Regarding the three long-slit spectra obtained of II Zw 40, the FWHM's measured along the spatial direction give values of $11^{\prime \prime} 67,11^{\prime \prime} 03$ and $11^{\prime \prime} 22$, somewhat larger than the average MIR seeing at the time of the observation (1'.11, 0.'91 and 0.'96, respectively). II Zw 40 is then marginally resolved but the slit is wide enough (1'.2) to ensure that practically all the MIR flux emitted is registered. This is so since in the last two cases the FWHM is similar to or smaller than the width of the slit and the flux level of all three spectra agrees well within the errors.

For He 2-10 A, the FWHM's of the two spectra which were obtained are 1". 83 and 1".37, greater than the respective MIR seeings $(0.77$ and 0.83$)$ and the slit width. Assuming that the source is Gaussian and that the slit is perfectly centred on the source peak, this implies that the slit might be registering about $60-75 \%$ of the total flux emitted by component $\mathrm{A}$. In the case of $\mathrm{He} 2-10 \mathrm{C}$, the three individual observations obtained give $F W H M$ 's of 1 '.22, 1 .'13 and 1.'47, the respective MIR seeings being $11^{\prime \prime} 0,0.28$ and 1 .' 19 . This source is then only slightly resolved. As for II Zw 40, we are confident that the slit is registering most of the MIR flux emitted by component $\mathrm{C}$ in all three cases.

The spectra were extracted through apertures of about $2 \times$ FWHM centred on the peak positions.

Line fluxes were measured by fitting a Gaussian and their quoted uncertainties (of the order of 5-20\%) include the statistical error associated with each point at a given wavelength. Upper limits are defined as the flux of a feature with a peak flux three times the continuum rms noise and a width equal to the instrumental resolution element.

\footnotetext{
1 www. ls.eso.org/lasilla/sciops/3p6/timmi
} 


\section{Results}

Here we present our high spatial resolution spectroscopy data using TIMMI2. First, a general description of each object is provided, followed by a description of the spectra in terms of general shape and line and molecular content. Our data is compared with observations reported in the literature.

A variety of fine-structure lines and broad-band dust features fall within the $N$-band spectroscopic range. The most relevant lines are [Ar III] at $9.0 \mu \mathrm{m}$, [S IV] at $10.5 \mu \mathrm{m}$ and [Ne II] at $12.8 \mu \mathrm{m}$. These lines require hard radiation with energies between $\sim 21$ and $35 \mathrm{eV}$, and the most likely explanation for their excitation mechanism is photoionisation by hot stars. In terms of molecular features, the MIR spectra of many starbursts are dominated by the well-known emission features at $8.6,11.2$ and $12.7 \mu \mathrm{m}$, commonly called the unidentified infrared (UIR) bands and now generally attributed to vibrational emission of Polycyclic Aromatic Hydrocarbons (PAHs) containing $\simeq 50$ carbon atoms (e.g. Allamandola et al. 1989; Puget \& Léger 1989; Peeters et al. 2004a). A silicate band centred around $9.7 \mu \mathrm{m}$ can also be present.

\subsection{NGC 3256}

\subsubsection{General description}

The galaxy is a peculiar spiral galaxy located at a distance of $37 \mathrm{Mpc}$ (Lípari et al. 2000). The angular scale at this distance is $1^{\prime \prime}=179 \mathrm{pc}$. This galaxy consists of a main body of about $60^{\prime \prime}$, two extended tidal tails that can be traced as far as $8^{\prime}$ and two faint external loops (Lípari et al. 2000). The central $\left(\sim 30^{\prime \prime}\right)$ region shows a knotty structure with a very unusual triple asymmetrical spiral arm morphology. The double tidal tails are characteristic of an interaction between two spiral galaxies of comparable mass (e.g. de Vaucouleurs \& de Vaucouleurs 1961).

High spatial resolution near-IR (e.g. Moorwood \& Oliva 1994; Kotilainen et al. 1996), MIR (Böker et al. 1997) and radio observations (e.g. Norris \& Forbes 1995; Neff et al. 2003) reveal two distinct nuclei aligned in the north-south direction and separated by $5^{\prime \prime}$. The southern nucleus has no optical counterpart due to the high extinction in this region. The presence of these two nuclei, which at radio wavelengths have approximately similar size, brightness and spectral index, suggests that the merger is not yet completed. This is supported by the $K^{\prime}$ surface brightness profile over the central $\left.15 \mathrm{kpc}(\sim 1)^{\prime} 4\right)$, which is clearly not that of a galaxy that has already relaxed sufficiently to be classified as elliptical (Moorwood \& Oliva 1994). The radio spectral indices obtained for both nuclei ( -0.9; Norris \& Forbes 1995) are quite steep and indicate that the radio emission is dominated by synchrotron radiation from cosmic-ray electrons accelerated by supernovae and that the contribution to the radio emission from H II regions is negligible.

The starburst nature of the northern nucleus of NGC 3256 is indicated by different authors: 1) the $10 \mu \mathrm{m}$ emission extends over a region $\sim 4 \mathrm{kpc}\left(\sim 22^{\prime \prime}\right)$ across with most of it originating outside the central $\mathrm{kpc}\left(\sim 5^{\prime \prime} .6\right)$ (Graham et al. 1984). 2) Glass \& Moorwood (1985) show that the JHKL colours of the galaxy are indicative of a young starburst. 3) NGC 3256 is located in the same region as starburst galaxies in IR diagnostic diagrams that use PAH emission features as a diagnostic tool for the physical processes powering Galactic nuclei (AGN versus starburst), indicating that at MIR wavelengths starburst activity is the dominant energy source (Peeters et al. 2004b). 4) NGC 3256 also presents a prominent $3.3 \mu \mathrm{m}$ PAH feature

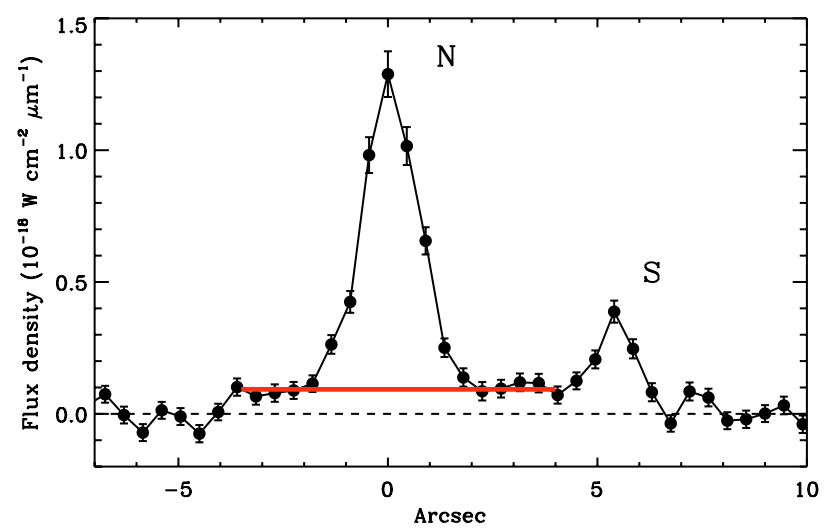

Fig. 1. Spatial variation of the [Ne II] line peak across the slit from north (left) to south (right). Error bars indicate $1 \sigma$ errors. A plateau which extends over $\sim 8^{\prime \prime}$ is indicated by a solid line in light colour. At the distance of NGC $3256,1^{\prime \prime}$ corresponds to $\sim 179 \mathrm{pc}$. The peak positions of the northern and southern nuclei are identified by the labels $\mathrm{N}$ and $\mathrm{S}$ respectively.

(Moorwood 1986) with an equivalent width typical of starburst galaxies. 5) Rowan-Robinson \& Crawford (1989) modelled the IRAS $12,25,60$ and $100 \mu \mathrm{m}$ fluxes and obtained that the contribution of a hidden AGN is $<5 \%$. 6) The specific search for the [Si VI] $1.96 \mu \mathrm{m}$ coronal line emission from a possibly obscured AGN proved negative (Moorwood \& Oliva 1994). 7) The central $3^{\prime \prime}$ of the nuclear region show strong recombination lines of hydrogen and helium and a prominent $\mathrm{CO}$ band absorption at $2.3 \mu \mathrm{m}$ (Doyon et al. 1994). These features provide further evidence for starburst activity since it implies the existence of a large number of OB stars and a young population of red supergiants. 8) The SWS spectrum of NGC 3256 shows no signatures of high-excitation lines common in galaxies powered by AGNs (Rigopoulou et al. 1996). This is confirmed by the high spatial resolution spectrum of the northern nucleus obtained by Siebenmorgen et al. (2004). The nature of the southern nucleus remains, however, unclear.

High spatial resolution (0.6) Chandra observations (Lira et al. 2002) find several (14) discrete sources embedded in a complex diffuse emission which contribute $\sim 20 \%$ of the total $\mathrm{X}$-ray emission in the $0.5-10 \mathrm{keV}$ energy range. Two of these discrete sources are coincident with the two nuclei, with the northern nucleus corresponding to the brightest X-ray source. These authors find no evidence for the presence of an AGN in the southern nucleus. However, based on the ratio of $6 \mathrm{~cm}$ radio emission to 2-10 keV X-ray emission, Neff et al. (2003) suggest the possibility that both nuclei might harbour lowluminosity AGNs generated in or fuelled by the galaxy merger. Nevertheless, based on HST STIS long-slit spectra of the northern nucleus, Lípari et al. (2004) suggest that the shape of the rotation curve and the emission-line profile can be explained by the presence of young star clusters with outflow in the core, and that most probably the associated compact X-ray emission and radio emission are the result of a few recent supernovae remnants.

\subsection{2. $N$-band spectra}

The spectral observations towards the nuclear region of NGC 3256 registered both the northern $(\mathrm{N})$ and southern (S) nuclei previously imaged at MIR wavelengths by Böker et al. (1997). Figure 1 shows the variation of the [Ne II] $12.8 \mu \mathrm{m}$ line peak flux across the slit from north to south. The northern 

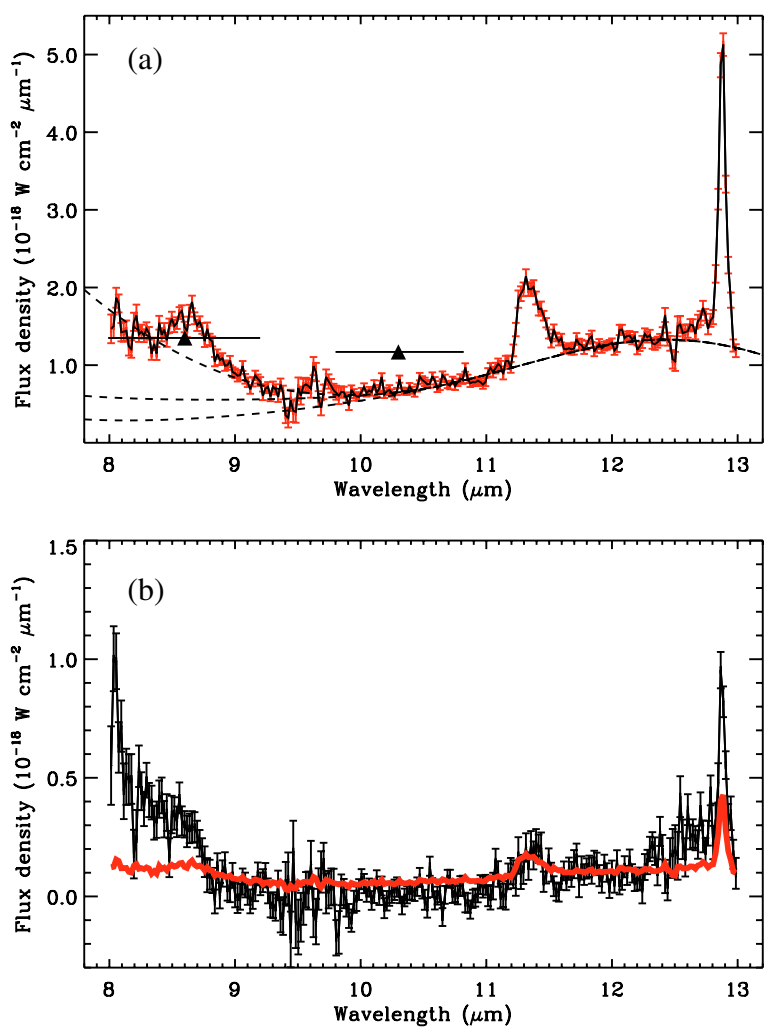

Fig. 2. a) $N$-band spectrum of the northern nucleus of NGC 3256 with $1 \sigma$ errors. The spectrum is characterised by a strong dust continuum, the 8.6, 11.2 and possibly the $12.7 \mu \mathrm{m}$ PAH emission bands and the atomic fine-structure line of [Ne II] at $12.8 \mu \mathrm{m}$. The dashed lines represent different local spline continua. The triangles indicate the TIMMI2 photometry in the 8.6 and $10.4 \mu \mathrm{m}$ filters obtained by Siebenmorgen et al. (2004). The FWHM's of these TIMMI2 filters are shown by horizontal bars. b) $\mathrm{N}$-band spectrum of the southern nucleus of NGC 3256 with $1 \sigma$ errors. The spectrum shows emission below $\sim 8.8 \mu \mathrm{m}$, a rising dust continuum after $\sim 11 \mu \mathrm{m}$, the $11.2 \mu \mathrm{m}$ PAH emission band and the fine-structure line of [Ne II] at $12.8 \mu \mathrm{m}$. For comparison, we show in light colour the spectrum of the northern nucleus scaled down by a factor of 12 .

nucleus has a peak flux of $(1.29 \pm 0.03) \times 10^{-18} \mathrm{~W} \mathrm{~cm}^{-2} \mu \mathrm{m}^{-1}$. The southern nucleus is much weaker, with a peak flux of $(0.39 \pm$ $0.03) \times 10^{-18} \mathrm{~W} \mathrm{~cm}^{-2} \mu \mathrm{m}^{-1}$. Faint [Ne II] emission was also detected extending about 4 " around the peak of NGC $3256 \mathrm{~N}$. This faint emission appears as an emission plateau with an average flux level of $(9.3 \pm 1.0) \times 10^{-20} \mathrm{~W} \mathrm{~cm}^{-2} \mu \mathrm{m}^{-1}$. This plateau coincides with a small spiral disc, at face-on position, which is a continuation of one of the spiral arms of the galaxy and reaches the very northern nucleus (Lípari et al. 2000).

The fact that NGC $3256 \mathrm{~N}$ is slightly resolved (see Sect. 2) allows for a comparison of the spatial variation of the [Ne II] line peak and other features such as the $11.2 \mu \mathrm{m}$ PAH peak emission and the continuum at $12 \mu \mathrm{m}$. No differences are evident, indicating that either the PDR and dust shell form a thin layer around the $\mathrm{N}$ nucleus or we are simply not resolving the emission.

Figure 2a shows the spectrum of NGC $3256 \mathrm{~N}$. Only the valid range between 8 and $13 \mu \mathrm{m}$ is plotted. The spectrum is characterised by a continuum due to warm dust, the 8.6, 11.2 and possibly also the $12.7 \mu \mathrm{m}$ PAH emission bands, and the atomic fine-structure line of [Ne II] at $12.8 \mu \mathrm{m}$. Siebenmorgen et al. (2004) have previously imaged the $\mathrm{N}$ nucleus at 8.6 and $10.4 \mu \mathrm{m}$ and obtained a TIMMI2 spectrum of this nucleus with a $3^{\prime \prime}$ slit. Their narrow-band filter photometry agrees well with

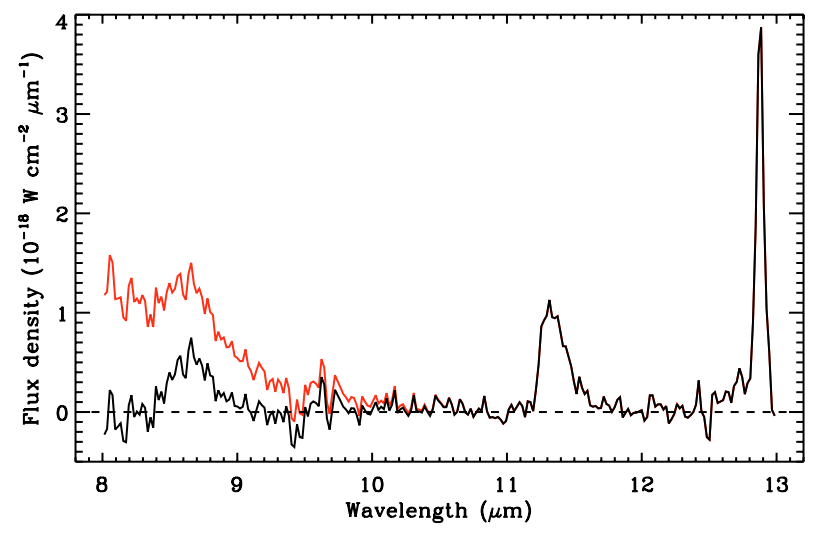

Fig. 3. Continuum subtracted spectrum of NGC 3256 N. The result of using the two extreme possibilities for the continuum plotted in Fig. 2 is shown by the dark and light lines. Note that the difference in both continua is the removal or not of the underlying PAH plateau and wing of the $7.7 \mu \mathrm{m}$ complex (see Fig. 4).

the flux level of our spectrum (cf. Fig. 2a). A comparison between the spectrum obtained by Siebenmorgen et al. (2004) and ours in terms of line and PAH band fluxes is done in Sects. 3.1.3 and 3.1.4.

Emission towards the southern nucleus was only detected shortwards of $\sim 8.8 \mu \mathrm{m}$ and beyond $11 \mu \mathrm{m}$. The spectrum of NGC $3256 \mathrm{~S}$ is shown in Fig. 2b. Like NGC 3256 N, the spectrum shows the $11.2 \mu \mathrm{m}$ PAH emission band, possibly also the 8.6 and 12.7 PAH bands, and the atomic fine-structure line of [Ne II] at $12.8 \mu \mathrm{m}$. A comparison with the spectrum of the $\mathrm{N}$ nucleus is also shown. Both spectra are similar, although with noticeable differences in the emission shortwards of $\sim 8.8 \mu \mathrm{m}$ and beyond $\sim 12 \mu \mathrm{m}$.

Graham et al. (1984) measured a $10 \mu \mathrm{m}$ flux of $(5.1 \pm 0.4) \times$ $10^{-18} \mathrm{~W} \mathrm{~cm}^{-2} \mu \mathrm{m}^{-1}$ in a $15^{\prime \prime}$ aperture, about 8 times larger than the flux we obtain at this wavelength towards the $\mathrm{N}$ nucleus, indicating that diffuse MIR emission exists around the two nuclei (cf. Sect. 3.1.1).

\subsubsection{Line fluxes}

Line fluxes towards NGC $3256 \mathrm{~N}$ and S are listed in Table 1. Only the line of [Ne II] at $12.8 \mu \mathrm{m}$ was detected. Upper limits to the fluxes of the [Ar III] 9.0 and [S IV] $10.5 \mu \mathrm{m}$ lines are also given. Considering the slit losses that might be affecting the [Ne II] line flux emitted by NGC $3256 \mathrm{~N}$ (about 32\%, see Sect. 2), the total [Ne II] line flux would be $\sim 31 \times$ $10^{-20} \mathrm{~W} \mathrm{~cm}^{-2}$.

Table 1 compares the fluxes observed towards the $\mathrm{N}$ and S nuclei with those observed by the large ISO/SWS beam and other apertures/slits. The ISO/SWS aperture is approximately $14^{\prime \prime} \times 20^{\prime \prime}$ up to $12 \mu \mathrm{m}$ and $14^{\prime \prime} \times 27^{\prime \prime}$ between 12 and $19.6 \mu \mathrm{m}$. The [Ar III] line flux measured by ISO is approximately 4 times larger than our upper limit, while the ISO [S IV] flux is too small to be detected by TIMMI2. Finally, the ISO [Ne II] line flux is about 3 times larger than the combined flux emitted by the $\mathrm{N}$ and $\mathrm{S}$ nuclei. This indicates that other objects besides the two nuclei must contribute strongly to the [Ar III] and [Ne II] line fluxes measured by ISO.

Siebenmorgen et al. (2004) only present the spectrum of the northern nucleus and do not comment on the detection of the southern one, probably due to the use of a wider slit ( $\left.3^{\prime \prime}\right)$. Their published spectrum most likely includes the contributions 
Table 1. Line fluxes and $3 \sigma$ upper limits measured towards the two nuclei in NGC 3256. Also included are line fluxes measured within other aperture/slits.

\begin{tabular}{lccccc}
\hline \hline Line & $\begin{array}{c}\lambda \\
(\mu \mathrm{m})\end{array}$ & $\begin{array}{c}\text { North } \\
\left(10^{-20} \mathrm{~W} \mathrm{~cm}^{-2}\right)\end{array}$ & $\begin{array}{c}\text { Sperture } \\
\left({ }^{\prime \prime}\right)\end{array}$ & Ref. \\
\hline$[\mathrm{Ar}$ III $]$ & 9.0 & $<1.2$ & $<1.2$ & 1.2 & 1 \\
{$[\mathrm{~S} \mathrm{IV}]$} & 10.5 & $<1.1$ & $<1.1$ & 1.2 & 1 \\
{$[\mathrm{Ne}$ II $]$} & 12.8 & $21 \pm 1^{\star}$ & $3.3 \pm 0.6$ & 1.2 & 1 \\
{$[\mathrm{~S} \mathrm{IV}]$} & 10.5 & $<1.6^{\diamond}$ & & 3 & 2 \\
{$[\mathrm{Ne}$ II $]$} & 12.8 & $28.5 \pm 1.0^{\diamond}$ & 3 & 2 \\
{$[\mathrm{Ne}$ II $]$} & 12.8 & $38^{\diamond}$ & 4.2 & 3 \\
{$[\mathrm{Ar}$ III $]$} & 9.0 & $4.7 \pm 0.4^{\Delta}$ & $14 \times 20$ & 4 \\
{$[\mathrm{~S} \mathrm{IV}]$} & 10.5 & $0.9 \pm 0.1^{\triangleleft}$ & $14 \times 20$ & 4 \\
{$[\mathrm{Ne}$ II $]$} & 12.8 & $89 \pm 14^{\Delta}$ & $14 \times 27$ & 4 \\
\hline
\end{tabular}

* When correcting for slit losses, we obtain a total line flux of about $31 \times 10^{-20} \mathrm{~W} \mathrm{~cm}^{-2}$ (cf. Sect. 3.1.3.).

$\checkmark$ This observation is probably centred on the $\mathrm{N}$ nucleus.

$\triangle$ The ISO/SWS aperture includes both $\mathrm{N}$ and $\mathrm{S}$ nuclei and any other possible mid-IR emitters. Errors are calculated from the typical ISO/SWS $1 \sigma$ absolute flux accuracy (Peeters et al. 2002b).

(1) This work; (2) Siebenmorgen et al. (2004); (3) Roche et al. (1991); (4) Verma et al. (2003).

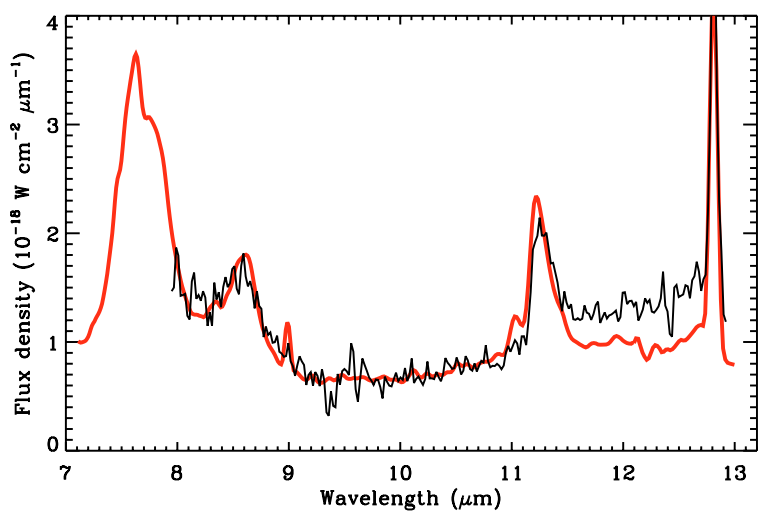

Fig. 4. Comparison between the $N$-band spectrum of NGC $3256 \mathrm{~N}$ (black line) and the ISO/SWS spectrum of the Galactic H II region IRAS $23133+6050$ convolved to the resolution $(\lambda / \Delta \lambda \sim 160)$ of TIMMI2 (light colour). The flux level of IRAS $23133+6050$ have been scaled down by a factor of 48 . The wavelength array of NGC $3256 \mathrm{~N}$ has been shifted by $-0.065 \mu \mathrm{m}$ in order to match the peak positions of the PAHs and [Ne II] line in the spectrum of IRAS 23133+6050.

of both nuclei. They estimate a [Ne II] line flux of $(28.5 \pm 1.0) \times$ $10^{-20} \mathrm{~W} \mathrm{~cm} \mathrm{~cm}^{-2}$, in good agreement with the value we obtain when the fluxes of both nuclei are added together and slit losses are considered. Integrated over an aperture of 4 ". 2 , Roche et al. (1991) measured a [Ne II] line flux of $38 \times 10^{-20} \mathrm{~W} \mathrm{~cm}^{-2}$, only slightly higher than the TIMMI2 line flux.

\subsubsection{PAH bands}

Both nuclei exhibit PAH emission features (see Fig. 2). The northern nucleus clearly shows the 8.6 and $11.2 \mu \mathrm{m}$ PAH bands, possibly the $12.7 \mu \mathrm{m}$ PAH band, the red wing of the $7.7 \mu \mathrm{m}$ PAH complex and that of the broad emission plateau underlying both the $7.7 \mu \mathrm{m}$ complex and the $8.6 \mu \mathrm{m}$ band, clearly seen shortwards of $\sim 9 \mu \mathrm{m}$ (see Fig. 4 for a comparison with the ISO/SWS spectrum of the Galactic H II region IRAS $23133+6050$ and e.g. the review by Peeters et al. 2004a). The southern nucleus shows the $11.2 \mu \mathrm{m}$ band and possibly also the 8.6 and $12.7 \mu \mathrm{m}$ PAH bands.
Table 2. Continuum subtracted 8.6, 11.2 and $12.7 \mu \mathrm{m}$ PAH band fluxes (in units of $10^{-20} \mathrm{~W} \mathrm{~cm}^{-2}$ ) measured towards the northern and southern nuclei. These are compared with other observations at different apertures.

\begin{tabular}{ccccc}
\hline \hline PAH band & $\begin{array}{c}\text { North } \\
\left(10^{-20} \mathrm{~W} \mathrm{~cm}^{-2}\right)\end{array}$ & $\begin{array}{c}\text { South } \\
\left({ }^{\prime \prime}\right)\end{array}$ & $\begin{array}{c}\text { Aperture } \\
\text { Ref. }\end{array}$ \\
\hline 8.6 & $18 \pm 4$ & - & 1.2 & 1 \\
11.2 & $25 \pm 4$ & $5 \pm 2$ & 1.2 & 1 \\
12.7 & $<13$ & $<7$ & 1.2 & 1 \\
11.2 & 30 & - & 3 & 2 \\
11.2 & $32^{\star}$ & - & 4.2 & 3 \\
11.2 & \multicolumn{2}{c}{$107^{\sharp}$} & 24 & 2 \\
\hline
\end{tabular}

* Although it is not indicated by the authors, we assume that these observations are centred on the $\mathrm{N}$ nucleus.

\# The ISOPHOT aperture includes both $\mathrm{N}$ and $\mathrm{S}$ nuclei and any other possible mid-IR emitters.

(1) This work; (2) Siebenmorgen et al. (2004); (3) Roche et al. (1991).

The PAH fluxes are determined by subtracting a local spline continuum (e.g. Fig. 2) and are given in Table 2. The presence of the $12.7 \mu \mathrm{m}$ band is highly dependent on the choice of the continuum which, in turn, is influenced by the flux level at the longest wavelengths (around $13 \mu \mathrm{m}$ ). Therefore, its detection is merely tentative and thus we simply give an upper limit.

The 11.2 PAH flux we obtain towards the $\mathrm{N}$ nucleus agrees well with those obtained through slightly larger apertures $\left(3^{\prime \prime}-4^{\prime \prime}\right.$, see Table 2). However, when compared with the 11.2 PAH flux measured by the large ISOPHOT aperture, the combined flux of both the $\mathrm{N}$ and $\mathrm{S}$ nuclei accounts only for $30 \%$ of the total. This is roughly the same contribution of the [Ne II] flux emitted by both nuclei to the total ISO/SWS [Ne II] flux. This suggests that this non-nuclear emission comes from $\mathrm{H}$ II regions and their associated PDRs.

The comparison of the PAH emission bands in both nuclei (see Fig. 2) reveals large differences in both the relative strength of the 11.2/12.7 PAH band ratio (assuming for the moment a positive detection of the $12.7 \mu \mathrm{m}$ band in both nuclei) and the plateau/11.2 ratio. In both cases, the southern nucleus has a stronger 12.7 PAH band and a stronger plateau both relative to the $11.2 \mu \mathrm{m}$ PAH band compared to that of the northern nucleus. Possible origins for this are the following. (i) A different PAH charge balance in both nuclei. Indeed, a larger fraction of ionic PAHs give rise to stronger emission in the 5-10 $\mu \mathrm{m}$ region (e.g. Peeters et al. 2002a) and hence might explain the difference in plateau/11.2 ratio. For the $12.7 \mu \mathrm{m}$ PAH, it is unclear at this moment whether it is due to neutral or cationic PAHs (Hony et al. 2001). (ii) The 12.7/11.2 ratio is a tracer of the PAH molecular edge structure (Hony et al. 2001). In this case, the S nucleus should have more irregular PAHs (Hony et al. 2001) likely due to a stronger radiation field and lower density. Molecular edge structure has little influence on the $\mathrm{CC}$ stretching and $\mathrm{CH}$ inplane-bending modes emitting in the $6-9 \mu \mathrm{m}$ region. (iii) The underlying plateau is believed to arise from larger PAH-related molecules or complexes (Allamandola et al. 1989) indicating that both nuclei might have a different PAH size distribution. As with the other two possibilities, it cannot explain at the same time the relative intensities of all PAH ratios.

\subsubsection{Extinction}

The extinction suffered by the line emission from NGC $3256 \mathrm{~N}$ has been estimated by Doyon et al. (1994) using two different 
methods: 1) the ratio of $\mathrm{Pa} \beta$ to $\mathrm{Br} \gamma$, which gives an absolute extinction at $2.2 \mu \mathrm{m}$ of $A_{\mathrm{K}}=0.55 \pm 0.09 \mathrm{mag}$; and 2) the ratio of [Fe II] 1.257 to [Fe II] $1.644 \mu \mathrm{m}$, which implies $A_{\mathrm{K}}=0.7 \pm$ 0.3 mag. Kotilainen et al. (1996) proposed a third method, which assumes the correlation between $6 \mathrm{~cm}$ radio and [Fe II] luminosities established by Forbes \& Ward (1993). They obtain an extinction of $A_{\mathrm{K}}=0.51$ for the $\mathrm{N}$ nucleus and of $A_{\mathrm{K}}=1.1$ for the $\mathrm{S}$ nucleus. For the $\mathrm{N}$ nucleus, the average of the three methods gives $A_{\mathrm{K}}=0.6$. These values of the extinction in the near-IR, i.e. $A_{\mathrm{K}}(\mathrm{N})=0.6$ and $A_{\mathrm{K}}(\mathrm{S})=1.1$, translates into $A_{\mathrm{V}} \sim 5.6$ and 10.2 mag for the $\mathrm{N}$ and $\mathrm{S}$ nuclei, respectively, assuming $R_{\mathrm{V}}=3.1$ (Mathis 1990). Based on the $H-K$ colours, Lípari et al. (2000) find $A_{\mathrm{V}}(\mathrm{N}) \sim 5.5$ and $A_{\mathrm{V}}(\mathrm{S}) \sim 16$ mag.

The extrapolation of these values of the extinction in the optical and near-IR to the MIR regime is not direct unless the exact shape and depth of the silicate absorption feature at $9.7 \mu \mathrm{m}$ is known. This could be done by fitting the dust continuum (cf. Martín-Hernández et al. 2005). However, the presence of the strong PAH bands hampers this task considerably. By simply assuming that the extinction law in the MIR can be described by the commonly used "astronomical silicate" (cf. Draine \& Lee 1984; Draine 1985), we obtain that $A_{12.8}=0.33 A_{\text {sil }}$ (Martín-Hernández et al. 2003), where $A_{12.8}$ is the extinction at the [Ne II] line central wavelength and $A_{\text {sil }}$ is the extinction at the peak of the silicate absorption. Assuming a ratio $A_{\mathrm{V}} / A_{\text {sil }} \sim 18.5$, found by Roche \& Aitken (1984) for the local diffuse interstellar medium, we obtain $A_{\text {sil }}(\mathrm{N}) \sim 0.4$ and $A_{\text {sil }}(\mathrm{S}) \sim$ 0.6 mag when the values above mentioned for the V-extinction towards the $\mathrm{N}$ and $\mathrm{S}$ nuclei (5.6 and 10.2) are used. Hence, we obtain $A_{12.8}(\mathrm{~N}) \sim 0.1$ and $A_{12.8}(\mathrm{~S}) \sim 0.2 \mathrm{mag}$. These values are to be considered as only rough estimates of the extinction affecting the [Ne II] line. When applying these extinction values to the [Ne II] line fluxes measured for both nuclei, we obtain $\sim 23 \times 10^{-20}$ and $\sim 4 \times 10^{-20} \mathrm{~W} \mathrm{~cm}^{-2}$, respectively. These fluxes are still within the $2 \sigma$ error bars of the observed fluxes. Even in the case of a larger extinction towards the $\mathrm{S}$ nucleus (16 mag, see above), the [Ne II] extinction corrected flux will be $\sim 4.3 \times$ $10^{-20}$, only $30 \%$ larger than the observed flux.

\subsubsection{Ionic abundances}

Ionic abundances can be determined from the measured strengths of the fine-structure and H I recombination lines. The ionic abundance of a certain ion $X^{+\mathrm{i}}$ with respect to hydrogen $\left(X^{+\mathrm{i}} / \mathrm{H}^{+}\right)$can be determined using the following expression (e.g. Rubin et al. 1988):

$\frac{X^{+\mathrm{i}}}{\mathrm{H}^{+}}=\frac{F_{X^{+\mathrm{i}}} / F_{\mathrm{HI}}}{\epsilon_{X^{+\mathrm{i}}} / \epsilon_{\mathrm{HI}}}$

where $F_{X^{+\mathrm{i}}}$ and $F_{\mathrm{HI}}$ are the extinction-corrected fluxes of any line produced by $X^{+\mathrm{i}}$ and $\mathrm{HI}$, and $\epsilon_{X^{+i}}$ and $\epsilon_{\mathrm{HI}}$ are their respective emission coefficients. This expression assumes that 1) the nebula is homogeneous with constant electron temperature and density; 2) all the line photons emitted in the nebula escape without absorption and therefore without causing further upward transitions; and 3) the volume occupied by $X^{+\mathrm{i}}$ and $\mathrm{H}^{+}$is the same. The fine-structure line emission coefficients depend on the electron temperature $\left(T_{\mathrm{e}}\right)$, density $\left(n_{\mathrm{e}}\right)$ and relevant atomic parameters (cf. Martín-Hernández et al. 2002). The H I emission coefficients can be derived by using the program INTRAT by Storey \& Hummer (1995).

In the case of NGC 3256, we can determine the abundance of $\mathrm{Ne}^{+}$with respect to $\mathrm{H}^{+}$. Adopting $T_{\mathrm{e}}=6000 \mathrm{~K}$
(Aguero \& Lipari 1991; Storchi-Bergmann et al. 1995; Lípari et al. 2000) and $n_{\mathrm{e}}=10^{3} \mathrm{~cm}^{-3}$ (Lípari et al. 2000) for both the $\mathrm{N}$ and $\mathrm{S}$ nuclei, we obtain $\epsilon_{[\mathrm{NeII}] 12.8}=9.95 \times 10^{-22}$ and $\epsilon_{\mathrm{Br} \gamma}=5.98 \times 10^{-27} \mathrm{erg} \mathrm{cm}^{-3} \mathrm{~s}^{-1}$. Kotilainen et al. (1996) measured the Br $\gamma$ flux within $3^{\prime \prime}$ apertures centred on both nuclei. They obtain de-reddened fluxes of $0.52 \times 10^{-20}$ and $0.14 \times$ $10^{-20} \mathrm{~W} \mathrm{~cm}^{-2}$ respectively for the $\mathrm{N}$ and $\mathrm{S}$ nuclei. We note that the $\mathrm{Br} \gamma$ line fluxes measured by Doyon et al. (1994), integrated over a square aperture of 3 .' $5 \times 3$.' 5 , are roughly twice those obtained by Kotilainen et al. (1996). However, we consider here the fluxes by Kotilainen et al. since their $\mathrm{Br} \gamma$ flux towards the $\mathrm{N} \mathrm{nu}-$ cleus predicts well the extinction corrected $\mathrm{H} \beta$ flux measured by Lípari et al. (2000). We will consider a standard $20 \%$ uncertainty for these line fluxes.

Using the [Ne II] line fluxes listed in Table 1, we obtain $\mathrm{Ne}^{+} / \mathrm{H}^{+}(\mathrm{N})=[2.4(2.6) \pm 0.5] \times 10^{-4}$ and $\mathrm{Ne}^{+} / \mathrm{H}^{+}(\mathrm{S})=$ $[1.4(1.7) \pm 0.5] \times 10^{-4}$, where the values in brackets are obtained by using the extinction-corrected [Ne II] line fluxes given in Sect. 3.1.5. The use of the Br $\gamma$ flux measured by Doyon et al. (1994) will give $\mathrm{Ne}^{+}$abundances twice as large. There also exists the fact that the [Ne II] and $\mathrm{Br} \gamma$ lines are not measured on the same aperture. In principle, one could be over/underestimating the Bry flux associated to the MIR sources. This uncertainty is, however, difficult to quantify.

Formally, the above computed $\mathrm{Ne}^{+}$abundances are lower limits to the $\mathrm{Ne}$ abundance: $\mathrm{Ne} / \mathrm{H}(\mathrm{N}) \gtrsim[2.0(2.2) \pm 0.4] \times$ $[\mathrm{Ne} / \mathrm{H}]_{\odot}$ and $\mathrm{Ne} / \mathrm{H}(\mathrm{S}) \gtrsim[1.2(1.7) \pm 0.4] \times[\mathrm{Ne} / \mathrm{H}]_{\odot}$. These limits agree with previous optical determinations (Lípari et al. 2000; Aguero \& Lipari 1991; Moran et al. 1999) which, using different calibrations, find oxygen abundances of 1.3 solar or even 2.6-3 times solar (assuming $12+\log (\mathrm{O} / \mathrm{H})_{\odot}=8.69$, Allende Prieto et al. 2001). However, several uncertainties affect the oxygen abundances derived from emission lines using the "strong line method" especially at high metallicity (e.g. Pilyugin 2001; Stasińska 2005). Furthermore, if the latest upward revision of the solar Ne abundance by $\sim 0.4-0.5$ dex suggested from helioseismology (Antia \& Basu 2005; Bahcall et al. 2005) and from solar type stars (Drake \& Testa 2005) is confirmed, our $\mathrm{Ne}$ abundance estimate could well be compatible with solar or even slightly sub-solar.

\section{2. $I I Z w 40$}

\subsubsection{General description}

II Zw 40 is at a distance of 9.2 Mpc (Vacca \& Conti 1992). This distance accounts for Galactic rotation but no Virgocentric flow, and assumes $H_{0}=75 \mathrm{~km} \mathrm{~s}^{-1} \mathrm{Mpc}^{-1}$. At this distance, $1^{\prime \prime}$ corresponds to $44.6 \mathrm{pc}$.

This compact galaxy consists of a bright core only $3^{\prime \prime} \times 5^{\prime \prime}$ in size with two faint and diffuse tails extending about $30^{\prime \prime}$ to the south-east (Sargent \& Searle 1970). Baldwin et al. (1982) carried out an emission-line optical study of the morphology and kinematics of II Zw 40. They considered that the two tails are reminiscent of interacting galaxies and suggested a possible collision between two small systems as the source of the present starburst.

II Zw 40 is one of the prototypes of the class of dwarf galaxies known as "detached extra-galactic H II regions" (Sargent \& Searle 1970). These systems are characterised by emission-line spectra (e.g. Walsh \& Roy 1993) similar to those of individual giant H II regions superimposed on a continuum which appears to be mainly due to O stars. The presence of about 200 WR stars 


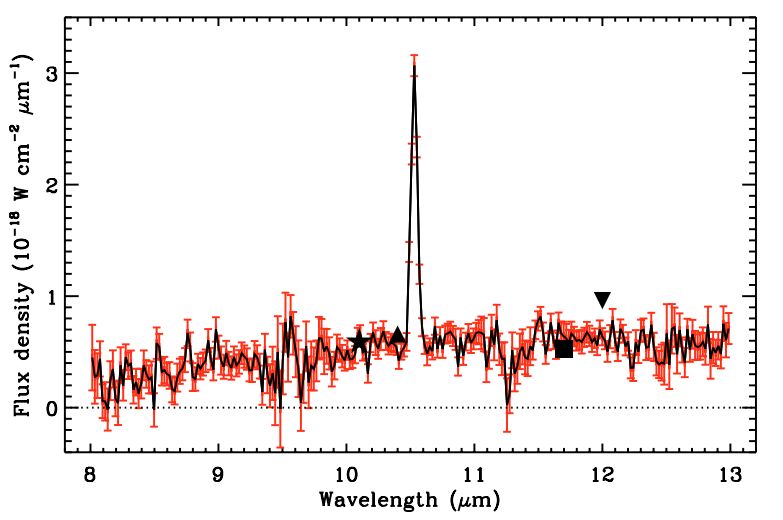

Fig. 5. $N$-band spectrum of the infrared supernebula in II Zw 40 with $1 \sigma$ errors. The spectrum is characterised by a rather flat continuum and a strong [S IV] $10.5 \mu \mathrm{m}$ line. For comparison, we plot the $10.1 \mu \mathrm{m}$ flux measured by Wynn-Williams \& Becklin (1986) in a 7'.7 aperture (star), the $10.4 \mu \mathrm{m}$ flux measured by Rieke \& Low (1972) in a 6" aperture (triangle), and the IRAS $12 \mu \mathrm{m}$ flux (upside down triangle; Vader et al. 1993). All these are broad band (filter $F W H M \sim 5 \mu \mathrm{m}$ ) photometric fluxes. The narrow band filter $11.7 \mu$ m flux measured by Beck et al. (2002), with a $F W H M$ of $\sim 1 \mu \mathrm{m}$, is indicated by a square.

is inferred from the flux of the He II $4684 \AA$ line (cf. Vacca \& Conti 1992).

The radio continuum emission of II Zw 40 is relatively compact and coincides with the optical bright core (e.g. Wynn-Williams \& Becklin 1986; Sramek \& Weedman 1986; Klein et al. 1991; Deeg et al. 1993; Beck et al. 2002). The spectral index of the overall radio emission has been discussed at length. It seems that the total $\mathrm{cm}$-wave emission of II Zw 40 is mostly thermal, with at most $30 \%$ of the total radio emission attributed to non-thermal processes (e.g. Sramek \& Weedman 1986; Joy \& Lester 1988). Within the central $6^{\prime \prime}$, the observed spectral index equals or exceeds the value for optically thin freefree emission ( $\alpha \gtrsim-0.1$, with $\left.S_{v} \propto v^{\alpha}\right)$, indicating no evidence for non-thermal emission from supernovae events and remnants. Further evidence of this lack of supernovae comes from the faintness of the [Fe II] line emission at $1.644 \mu \mathrm{m}$ (e.g. Vanzi et al. 1996), which is more likely to be produced in shocks than in photoionised gas. High-spatial resolution $\left(\sim 0{ }^{\prime} 1\right)$ imaging at $2 \mathrm{~cm}$ reveals a complex of smaller nebulae within the bright radio core (Beck et al. 2002).

II Zw 40 was first detected at $10 \mu \mathrm{m}$ by Rieke \& Low (1972). Later observations by Wynn-Williams \& Becklin (1986) revealed that most of the $10 \mu \mathrm{m}$ emission arises from a region less than $4^{\prime \prime}$ in diameter, more compact than the near-IR and optical emission. Recently, Keck MIR imaging has shown a bright source with a diameter $(F W H M)$ of about $0 .{ }^{\prime} 5$ coincident with the radio compact emission (Beck et al. 2002).

\subsection{2. $N$-band spectrum}

Figure 5 shows the spectrum of the infrared nebula in II Zw 40. Only the valid range between 8 and $13 \mu \mathrm{m}$ is plotted. The spectrum of II Zw 40 is characterised by a rather flat continuum and a strong [S IV] line at $10.4 \mu \mathrm{m}$.

II Zw 40 has been previously observed at MIR wavelengths using a wide range of apertures: at $10.1 \mu \mathrm{m}$ in a 7.17 aperture (Wynn-Williams \& Becklin 1986); at $10.4 \mu \mathrm{m}$ in a 6" aperture; at $11.7 \mu \mathrm{m}$, with a spatial resolution of $0.3-0.5$; and at $12 \mu \mathrm{m}$ with IRAS (Vader et al. 1993). These various photometric fluxes are indicated in Fig. 5 and show a perfect agreement with the
Table 3. Line fluxes and $3 \sigma$ upper limits measured towards II Zw 40. These are compared with measurements made with ISO/SWS.

\begin{tabular}{lcccc}
\hline \hline Line & $\begin{array}{c}\lambda \\
(\mu \mathrm{m})\end{array}$ & $\begin{array}{c}\text { Line Flux } \\
\left(10^{-20} \mathrm{~W} \mathrm{~cm}^{-2}\right)\end{array}$ & $\begin{array}{c}\text { Aperture } \\
\left({ }^{\prime \prime}\right)\end{array}$ & Ref. \\
\hline$[\mathrm{Ar}$ III $]$ & 9.0 & $<2.6$ & 1.2 & 1 \\
{$[\mathrm{~S} \mathrm{IV}]$} & 10.5 & $15.5 \pm 0.8$ & 1.2 & 1 \\
{$[\mathrm{Ne}$ II $]$} & 12.8 & $<2.2$ & 1.2 & 1 \\
{$[\mathrm{Ar}$ III $]$} & 9.0 & $2.0 \pm 0.2$ & $14 \times 20$ & 2 \\
{$[\mathrm{~S} \mathrm{IV}]$} & 10.5 & $20 \pm 2$ & $14 \times 20$ & 2 \\
{$[\mathrm{Ne}$ II $]$} & 12.8 & $1.7 \pm 0.3$ & $14 \times 27$ & 2 \\
\hline
\end{tabular}

(1) This work; (2) Verma et al. (2003).

spectrum we have obtained, even in the case of the IRAS $12 \mu \mathrm{m}$ flux measured with a beam that includes the entirely galaxy. This suggests that the MIR flux of the galaxy is confined to the compact $(0.5)$ infrared source we have observed with TIMMI 2.

\subsubsection{Line fluxes}

Towards II Zw 40, only the [S IV] line is detected. Its line flux and the upper limits of the non-detected lines are measured as described above (cf. Sect. 3.1.3) and listed in Table 3.

Table 3 compares the fluxes observed by TIMMI2 towards the infrared nebula in II Zw 40 with those observed by the large ISO/SWS aperture. The upper limits to the [Ar III] and [Ne II] line fluxes are consistent with the ISO values. Regarding [S IV], the ISO flux is only 1.3 times larger than the TIMMI 2 value. Hence, it seems valid to consider (as it has been stated in the previous section) that practically all of the midinfrared line emission emitted by the galaxy comes from this compact nebula.

\subsubsection{Extinction}

The analysis of the extinction towards II Zw 40 is complicated by the low galactic latitude of the galaxy, which implies some foreground extinction. Values of the extinction based on a foreground screen model and derived from the $\mathrm{H} \alpha / \mathrm{H} \beta, \mathrm{H} \alpha / \mathrm{Br} \gamma$ or $\mathrm{H} \beta / \mathrm{Br} \gamma$ line ratios are in the range $A_{\mathrm{V}}=2.1-3.0$ mag (e.g. Sargent \& Searle 1970; Wynn-Williams \& Becklin 1986; Vanzi et al. 1996; Joy \& Lester 1988; Davies et al. 1998). Walsh \& Roy (1993) present a map of the total (Galactic + extra-galactic) $A_{\mathrm{V}}$ derived from the $\mathrm{H} \alpha / \mathrm{H} \beta$ ratio. The map contains values of $A_{\mathrm{V}}$ as large as $4.5 \mathrm{mag}$, found preferentially in the northern half of the bright optical core of II Zw 40 with a general decrease to the south-east. However, non optical studies obtain much larger visual extinctions. Near-IR observations of II Zw 40 give $\mathrm{Br} \gamma$ fluxes of $(3.40-4.55) \times 10^{-21} \mathrm{~W} \mathrm{~cm}^{-2}$ measured in apertures between $3^{\prime \prime}$ and 9" (Joy \& Lester 1988; Ho et al. 1990; Vanzi et al. 1996; Davies et al. 1998; Coziol et al. 2001). Using this range in the observed $\mathrm{Br} \gamma$ flux, Beck et al. (2002) estimated an extinction of $A_{\mathrm{V}} \sim 8-10$ based on their $2 \mathrm{~cm}$ free-free flux from a region $\sim 3^{\prime \prime}$. A similar value for the visual extinction $\left(A_{\mathrm{V}} \sim 10 \mathrm{mag}\right.$ ) is found by Verma et al. (2003) based on ISO $\mathrm{H}$ I recombination lines. Other authors also argue (though from modelling of the SED) for a very high extinction $\left(A_{\mathrm{V}} \sim 20-30\right)$ for this object (Hunt et al. 2005). However, such a high extinction should lead to a significant silicate absorption band, which is not seen in the TIMMI2 spectrum (Fig. 5). Hence, unless the dust is very deficient in silicate, we do not favor such a high $A_{\mathrm{V}}$. Jaffe et al. (1978) propose a mixture of foreground and internal 
extinction and reproduce the Balmer decrement with foreground and internal extinctions of 0.8 and 8 mag respectively.

The extrapolation of the extinction in the optical and near-IR to the MIR regime is not direct. We can, however, estimate upper limits to the extinction that affects the MIR lines observed in II Zw 40 by adopting $A_{\mathrm{V}}=10 \mathrm{mag}\left(A_{\mathrm{K}}=1.1\right.$, Rieke \& Lebofsky 1985) and the method described in Sect. 3.1.5, i.e. using an extinction law defined by the "astronomical silicate" with $A_{\mathrm{V}} / A_{\text {sil }}=18.5$. We obtain $A_{9.0}=0.86 \times A_{\text {sil }}=0.46$, $A_{10.5}=0.80 \times A_{\text {sil }}=0.43, A_{12.8}=0.33 \times A_{\text {sil }}=0.18$, $A_{15.5}=0.27 \times A_{\text {sil }}=0.15, A_{18.7}=0.36 \times A_{\text {sil }}=0.19$ and $A_{25.9}=$ $0.19 \times A_{\text {sil }}=0.10 \mathrm{mag}$, where these are, respectively, the extinction at the central wavelengths of the [Ar III] 9.0, [S IV] 10.5, [Ne II] 12.8, [Ne III] 15.5, [S III] 18.7 and [O IV] $25.9 \mu \mathrm{m}$ lines (cf. Martín-Hernández et al. 2003). The extinction affecting the [Ar II] $7.0 \mu \mathrm{m}$ is obtained by simply extrapolating the value in the $K$-band considering a power law $A_{\lambda} \propto \lambda^{-1.7}$. We obtain $A_{7.0}=0.14 \times A_{\mathrm{K}}=0.15 \mathrm{mag}$.

These values of the extinction have to be considered as simply rough estimates and will be later considered in Sect. 4.4, where the stellar content of II Zw 40 will be discussed. Moreover, we might be overestimating the extinction for the lines around 10 and $18 \mu \mathrm{m}$ since, as we mention above, the TIMMI2 spectrum does not show evidence for silicate absorption.

\subsubsection{Ionic abundances}

Following Sect. 3.1.6, we can determine the ionic abundance of $\mathrm{S}^{3+}$ with respect to $\mathrm{H}^{+}$. Beck et al. (2002) predict an unextincted $\mathrm{Br} \gamma$ flux of $1.2 \times 10^{-20} \mathrm{~W} \mathrm{~cm}^{-2}$ based on radio continuum observations (see previous section). We will use this value hereafter and consider a standard $20 \%$ uncertainty.

Adopting $T_{\mathrm{e}}=12500 \mathrm{~K}$ (Vacca \& Conti 1992; Walsh \& Roy 1993; Pérez-Montero \& Díaz 2003) and $n_{\mathrm{e}}=1700 \mathrm{~cm}^{-3}$ (see

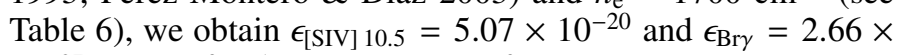
$10^{-27} \mathrm{erg} \mathrm{cm}^{-3} \mathrm{~s}^{-1}$. The resulting $\mathrm{S}^{3+} / \mathrm{H}^{+}$is $[6.8(10.0) \pm 2.0] \times$ $10^{-7}$, where the value in brackets corresponds to the abundance obtained by using the extinction corrected [S IV] line flux (cf. Sect. 3.2.4).

Measurements of the oxygen abundance towards II Zw 40 (e.g Vacca \& Conti 1992; Pagel et al. 1992; Walsh \& Roy 1993; Masegosa et al. 1994; Guseva et al. 2000; Pérez-Montero \& Díaz 2003) give an average value $\mathrm{O} / \mathrm{H} \sim 1 / 3[\mathrm{O} / \mathrm{H}]_{\odot}$. The value we obtain for $\mathrm{S}^{3+} / \mathrm{H}^{+}$is only about $20 \%$ of the sulphur abundance expected for a nebula with a metallicity $1 / 3$ of the solar metallicity. Most of the sulphur must be either in the form of $\mathrm{S}^{++}$or $\mathrm{S}^{4+}$.

Since it has been proven that practically all of the infrared emission of II Zw 40 comes from this compact core (e.g. Beck et al. 2002), we can estimate the amount of sulphur locked in the form of $\mathrm{S}^{++}$by using the [S III] $18.7 \mu \mathrm{m}$ line flux measured by ISO $\left(5.1 \pm 1.0 \times 10^{-20} \mathrm{~W} \mathrm{~cm}^{-2}\right.$; cf. Verma et al. 2003). Adopting the above values for the electron temperature and density, we obtain $\epsilon_{\text {SIII } 18.7}=1.28 \times 10^{-20} \mathrm{erg} \mathrm{cm}^{-3} \mathrm{~s}^{-1}$. The resulting $\mathrm{S}^{++} / \mathrm{H}^{+}$ is $[8.8(10.5) \pm 3.0] \times 10^{-7}$, where, again, the value in brackets is obtained by adopting the extinction estimated in the previous section. Hence, $\mathrm{S}^{++}$and $\mathrm{S}^{3+}$ account for less than $40 \%$ of the expected sulphur abundance. The rest must be in the form of $\mathrm{S}^{4+}$, which needs energies around $47 \mathrm{eV}$ in order to be produced. Unfortunately, lines of $\mathrm{S}^{4+}$ are not present in the infrared to prove this result but the line of [O IV] at $25.9 \mu \mathrm{m}$ has been observed by ISO (and the ionisation edge for the creation of this ion is $54.9 \mathrm{eV}$, even higher).
Larger values of the density will give larger $\mathrm{S}^{++} / \mathrm{H}^{+}$and $\mathrm{S}^{3+} / \mathrm{H}^{+}$abundances because collisional de-excitation begins to play an important role. However, densities much larger than $10^{4} \mathrm{~cm}^{-3}$ (we note that the critical densities of the [S III] 18.7 and [S IV] $10.5 \mu \mathrm{m}$ lines are, respectively, $10^{4}$ and $3.7 \times 10^{4} \mathrm{~cm}^{-3}$; see Martín-Hernández et al. 2002) are necessary in order to produce an important increase in these abundances. Such high densities are not probable in H II regions, even in ultracompact ones (e.g. Wood \& Churchwell 1989; Kurtz et al. 1994).

\subsection{Henize 2-10}

\subsubsection{General description}

The radial velocity of He $2-10\left(\sim 860-870 \mathrm{~km} \mathrm{~s}^{-1}\right)$ yields a distance anywhere from 6 to $14 \mathrm{Mpc}$ depending on the chosen value of $H_{0}$ and assumptions on the Virgocentric flow. As done by other authors (e.g. Vacca \& Conti 1992), we adopt a reasonable compromise of $9 \mathrm{Mpc}$, which accounts for Galactic rotation but no Virgocentric flow, and assumes $H_{0}=75 \mathrm{~km} \mathrm{~s}^{-1} \mathrm{Mpc}^{-1}$. At this distance, $1^{\prime \prime}$ corresponds to $43.6 \mathrm{pc}$.

He 2-10 was the first emission-line galaxy to exhibit evidence for WR stars (Allen et al. 1976) and it is considered the prototype for WR galaxies (Conti 1991). High-resolution observations in the optical and near-IR (see Cabanac et al. 2005) show a bright central nucleus, generally refered to as region A, resolved into several UV-bright super star clusters which lie in an arc of about 2" (Conti \& Vacca 1994). This central nucleus is surrounded by two presumably older star-forming regions, named B and C. Region B, to the east, shows a mixed population of blue and red clusters (only detected at wavelenghts longer than $2.2 \mu \mathrm{m}$ ). Region $\mathrm{C}$, on the northwest side, has a long tail containing bright red clusters as well.

VLA radio continuum imaging (Kobulnicky \& Johnson 1999; Johnson \& Kobulnicky 2003) reveals 5 compact $\sim 1 \mathrm{mJy}$ radio sources in the central region aligned in a east-west orientation. These radio sources are labeled 1 to 5 from west to east. While the global radio spectral index is highly nonthermal ( $\alpha \simeq-0.5$, with $S_{v} \propto v^{\alpha}$ ) and consistent with synchrotron radiation produced in supernova explosions, these compact radio sources have positive $(\alpha>0)$ spectral indices suggesting an optically thick thermal bremsstrahlung origin, consistent with unusually dense H II regions.

The presence and importance of dust in the central region was confirmed by high spatial resolution MIR images (Sauvage et al. 1997; Beck et al. 2001; Vacca et al. 2002). The spatial distribution of the IR emission (which extends less than $5^{\prime \prime}$ ) agrees strikingly with that of the radio continuum. In fact, the subarsecond resolution observations by Vacca et al. (2002) using the Gemini North Telescope were able to detect 4 of the 5 radio knots observed by Kobulnicky \& Johnson (1999). These MIR nebulae provide at least $80 \%$ of the total flux seen by IRAS at $12 \mu \mathrm{m}$ with a beam that included the entirely galaxy (Beck et al. 2001).

Recent high resolution observations in $K_{\mathrm{S}}(2.2 \mu \mathrm{m}), L^{\prime}$ (3.8 $\mu \mathrm{m})$ and $M^{\prime}(4.8 \mu \mathrm{m})$ bands by Cabanac et al. (2005) have provided a detailed explanation of the multiwavelength appearance of He 2-10 from the optical to the radio. Contrary to what it was thought previously, these authors show that practically all the radio knots can be associated with $K_{\mathrm{S}^{-}}$and $L^{\prime}$-emitting regions which implies a revision of their physical nature. These authors tentatively review the classification of the radio knots and only classify knots $1+2$ and 5 as bona fide ultradense H II regions. They have counterparts in the NIR but not in the 

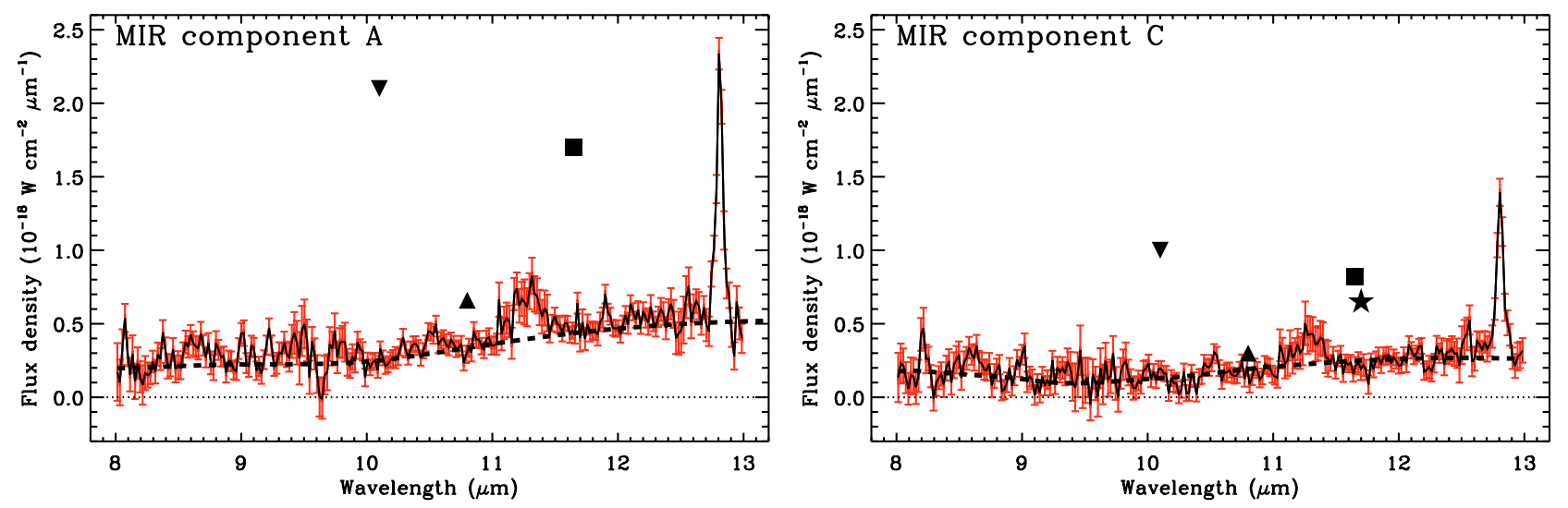

Fig. 6. $N$-band spectra of MIR components A (left) and C (right) in He 2-10 with $1 \sigma$ errors. The spectrum of A is roughly twice as bright as that of $\mathrm{C}$. Both spectra are very similar and are characterised by a rising dust continuum, weak PAH emission bands at 8.6 and $11.2 \mu \mathrm{m}$ and a strong [Ne II] $12.8 \mu \mathrm{m}$ line. For comparison, we plot the 10.1 and $11.65 \mu \mathrm{m}$ fluxes measured by Sauvage et al. (1997) for both components A and $\mathrm{C}$ (upside down triangle and square, respectively). The first one is a broad band measurement with a $F W H M \sim 5 \mu \mathrm{m}$, while the second one is a narrow band observation with a $F W H M$ of $\sim 1.7 \mu \mathrm{m}$. The narrow band $(F W H M \sim 1 \mu \mathrm{m}) 11.7 \mu \mathrm{m}$ flux measured by Beck et al. (2001) is plotted by a star. In the left panel, it coincides with the value quoted by Sauvage et al. (1997). The broad band $10.8 \mu \mathrm{m}$ fluxes measured by Vacca et al. (2002) for radio knots $1+2$ (C) and 4 (A) are plotted as a triangle. The dashed line represents the local spline continuum.

visible which implies a significant optical depth (typically $\gtrsim 10$ ) and thus a young age although possibly not as young as previoulsy postulated. Regarding knots 3 and 4, they propose that they are supernova remnants mixed with normal H II regions.

\subsection{2. $N$-band spectrum}

Our MIR observations were centred on the positions of the MIR counterparts to the radio knots 4 and $1+2$, named $\mathrm{A}$ and $\mathrm{C}$ respectively following the nomenclature by Beck et al. (2001) (see Sect. 3.3.1). These MIR regions A and C should not be mistaken with the equally named optical components (see previous section). Figure 6 shows the spectra of He 2-10 A and C. They show PAH emission bands at 8.6 and $11.2 \mu \mathrm{m}$ and the line of [Ne II] at $12.8 \mu \mathrm{m}$, while the presence of silicate in absorption is not clear. The presence of this feature in absorption is however more evident in the MIR spectrum of Phillips et al. (1984) obtained within a beam of 5 .' 9 .

High spatial observations of $\mathrm{He} 2-10$ have been done at 10.1 and $11.65 \mu \mathrm{m}$ by Sauvage et al. (1997), at $11.7 \mu \mathrm{m}$ by Beck et al. (2001) and at $10.8 \mu \mathrm{m}$ by Vacca et al. (2002). The photometric fluxes these authors have obtained are indicated in Fig. 6. While there is a huge discrepancy between our spectra and the fluxes obtained by Sauvage et al. (1997) and Beck et al. (2001), they agree rather well with those of Vacca et al. (2002). Possible discrepancies between these photometric measurements are largely discussed by Vacca et al. (2002). They could be due to differences in the $N$-band filter transmission profiles and central wavelengths, the use of a wrong colour term in the photometric calibration, non-photometric conditions during the observations or to a wrong background correction beneath the knots.

In the case of He 2-10 A, we have compared the spatial distribution of the [Ne II] and $11.2 \mu$ m peaks with that of the continuum at $12 \mu \mathrm{m}$. No differences are evident, consistent with the fact that the emission is only slightly resolved.

\subsubsection{Line fluxes}

We detect the [Ne II] line towards He 2-10 A and C. [Ne II] line fluxes and upper limits of the non-detected lines are measured as described in Sect. 3.1.3 and listed in Table 4.
Table 4. Line fluxes and $3 \sigma$ upper limits measured towards He 2-10. These are compared with fluxes measured using other apertures/slits.

\begin{tabular}{lccccc}
\hline \hline Line & $\begin{array}{c}\lambda \\
(\mu \mathrm{m})\end{array}$ & $\begin{array}{c}\mathrm{He} 2-10 \mathrm{~A} \\
\left(10^{-20} \mathrm{~W} \mathrm{~cm}^{-2}\right)\end{array}$ & $\begin{array}{c}\text { He 2-10 C } \\
\left({ }^{\prime \prime}\right)\end{array}$ & $\begin{array}{c}\text { Aperture } \\
\text { Ref. }\end{array}$ \\
\hline$[\mathrm{Ar}$ III $]$ & 9.0 & $<1.8$ & $<1.6$ & 1.2 & 1 \\
{$[\mathrm{~S} \mathrm{IV}]$} & 10.5 & $<1.1$ & $<1.3$ & 1.2 & 1 \\
{$[\mathrm{Ne}$ II $]$} & 12.8 & $12 \pm 1^{\star}$ & $6.8 \pm 0.5$ & 1.2 & 1 \\
{$[\mathrm{Ne}$ II $]$} & 12.8 & \multicolumn{2}{c}{$19.5 \pm 3.5$} & 5.9 & 2 \\
{$[\mathrm{Ne}$ II $]$} & 12.8 & \multicolumn{2}{c}{20} & 5.9 & 3 \\
{$[\mathrm{Ar}$ III $]$} & 9.0 & $1.6 \pm 0.5$ & - & $2.0 \mathrm{EW}$ & 4 \\
{$[\mathrm{~S} \mathrm{IV}]$} & 10.5 & $0.5 \pm 0.3$ & - & $1.6 \mathrm{EW}$ & 4 \\
{$[\mathrm{Ne}$ II $]$} & 12.8 & $20 \pm 2$ & - & $2.0 \mathrm{NS}$ & 4 \\
{$[\mathrm{Ne}$ II $]$} & 12.8 & $25 \pm 5$ & - & $1.6 \mathrm{EW}$ & 4 \\
\hline
\end{tabular}

* When correcting for slit losses, we obtain a total line flux of about $(16-20) \times 10^{-20} \mathrm{~W} \mathrm{~cm}^{-2}$ (cf. Sect. 3.3.3).

(1) This work; (2) Phillips et al. (1984); (3) Roche et al. (1991); (4) Beck et al. (1997).

Regarding He 2-10, spectra of its nuclear region have been previously obtained by Phillips et al. (1984), Roche et al. (1991) and Beck et al. (1997). A comparison between the fluxes obtained by these authors and ours is shown in Table 4. The [Ar III] and [S IV] line fluxes obtained by Beck et al. (1997) towards He 2-10 A agree well with our upper limits. Their [Ne II] line fluxes are, however, larger than the value we get. Still, slit losses might be affecting the [Ne II] line flux we measure. In Sect. 2, we estimate that our slit might only be registering about $60-75 \%$ of the total flux emitted by component A. Considering this loss, the total [Ne II] line flux would be about $(16-20) \times$ $10^{-20} \mathrm{~W} \mathrm{~cm}^{-2}$, a value that perfectly agrees with the fluxes obtained by Beck et al. using larger slit widths. The comparison with the [Ne II] flux obtained by Phillips et al. (1984) and Roche et al. (1991) is more complicated since they do not specify the central position of their apertures. However, it seems natural to assume that their apertures are centred on the brightest component A. Their [Ne II] fluxes would then agree well with the [Ne II] fluxes obtained by Beck et al. towards A and our value when corrected for slit losses. 
Table 5. Continuum subtracted 8.6 and $11.2 \mu \mathrm{m}$ PAH band fluxes (in units of $10^{-20} \mathrm{~W} \mathrm{~cm}^{-2}$ ) measured towards $\mathrm{He} 2-10 \mathrm{~A}$ and $\mathrm{C}$.

\begin{tabular}{lcccc}
\hline \hline Component & $8.6 \mu \mathrm{m}$ & $11.2 \mu \mathrm{m}$ & Aperture $\left(^{\prime \prime}\right)$ & Ref. \\
\hline A & $<5^{\triangle}$ & $9 \pm 5$ & 1.2 & 1 \\
C & $<7^{\triangle}$ & $6 \pm 5$ & 1.2 & 1 \\
$?$ & - & $12 \pm 2^{\natural}$ & 5.9 & 2 \\
\hline
\end{tabular}

${ }^{\Delta}$ Given as an upper limit due to the large error bars (see Fig. 6). ${ }^{\natural}$ The central position of this observation is uncertain. It is likely centred on component A.

(1) This work; (2) Phillips et al. (1984).

\subsubsection{PAH bands}

PAH emission bands at 8.6 and $11.2 \mu \mathrm{m}$, although very weak, have been detected in the spectra of $\mathrm{He} 2-10 \mathrm{~A}$ and $\mathrm{C}$. The PAH fluxes are determined by subtracting a local spline continuum (Fig. 6) and are listed in Table 5. The continuum subtracted spectra of both components are identical within the errors except for the [Ne II] line. The $11.2 \mu \mathrm{m}$ PAH flux has been previously measured towards the central 5.'9 of He 2-10 by Phillips et al. (1984). They quote a PAH band flux which is in reasonably agreement with the combined flux we obtain for components A and $\mathrm{C}$.

\subsubsection{Extinction}

The central region of $\mathrm{He} 2-10$ is a complex one and hence is not surprising that extinctions measured at different wavelengths do not agree well with one another. For instance, Vacca \& Conti (1992) measure $A_{\mathrm{v}} \sim 1.7$ from optical spectroscopy. Cabanac et al. (2005) derive $A_{\mathrm{v}}=1.25$ from the $\mathrm{H}_{\alpha} / \mathrm{Br} \gamma$ ratio and $A_{\mathrm{v}}=10.5$ from the $\operatorname{Br} \gamma / \mathrm{Br} 10$ ratio. From the $\operatorname{Br} \alpha / \operatorname{Br} \gamma$ line ratio observed by Kawara et al. (1989) one finds as well an extinction of $A_{\mathrm{v}} \sim 10 \mathrm{mag}$ which agrees well with the $15 \pm 5 \mathrm{mag}$ obtained by Phillips et al. (1984) from the depth of the silicate absorption. On the other hand, the beam-averaged $\mathrm{CO}$ column density towards the large optical starburst A implies an extinction of $\sim 30$ mag.

As mentioned in previous sections, the extrapolation of the extinction in the optical and near-IR to the MIR regime is not direct. Moreover, as in the case of NGC 3256 (cf. Sect. 3.1.5), the presence of PAH bands hampers the fitting of the dust continuum. Therefore, as it has been done previously, we will simply give rough estimates of the extinction in the MIR by using the "astronomical silicate" with $A_{\mathrm{V}} / A_{\text {sil }}=18.5$ (see Sect. 3.1.5) and considering $A_{\mathrm{V}}=10$. Using this, we have $A_{12.8}=0.33 A_{\text {sil }}=$ 0.2 mag. When applying this extinction value to the [Ne II] line fluxes measured for components $\mathrm{A}$ and $\mathrm{C}$, we obtain, respectively, $\sim 14 \times 10^{-20}$ and $\sim 8 \times 10^{-20} \mathrm{~W} \mathrm{~cm}^{-2}$.

\subsubsection{Ionic abundances}

As was done in Sect. 3.1.6, we can estimate the ionic abundance of $\mathrm{Ne}^{+}$with respect to $\mathrm{H}^{+}$.

Measurements of the $\operatorname{Br} \gamma$ line flux have only been obtained at low spatial resolutions, with apertures of, e.g. 7 ". $1 \times 3$ ". 5 (Kawara et al. 1989), 5" (Doyon et al. 1992) or 2'. $4 \times 15^{\prime \prime} .6$ (Vanzi \& Rieke 1997). Nevertheless, we can give estimates of the $\mathrm{Br} \gamma$ emission of knots $\mathrm{A}$ and $\mathrm{C}$ from the associated radio emission.

Johnson \& Kobulnicky (2003) provided 0.7 cm flux densities. Measurements at this frequency are likely to contain an insignificant non-thermal contribution and are usually optically thin. They measure flux densities of $2.91 \mathrm{mJy}$ for component A (equivalent to radio source \#4) and $1.87 \mathrm{mJy}$ for component $\mathrm{C}$ (by adding the contributions of radio sources \#1 and \#2). Adopting an electron temperature of 10000 K (Johansson 1987; Vacca \& Conti 1992) and the electron densities listed in Table 6 , we estimate $\mathrm{Br} \gamma$ fluxes of $0.41 \times 10^{-20}$ and $0.26 \times 10^{-20} \mathrm{~W} \mathrm{~cm}^{-2}$ for components $\mathrm{A}$ and $\mathrm{C}$ (we will consider a standard $20 \%$ uncertainty for these line fluxes). These values can be compared with the previously mentioned $\operatorname{Br} \gamma$ observations. These authors find Br $\gamma$ fluxes between $4.4 \times 10^{-21}$ and $6.5 \times 10^{-21} \mathrm{~W} \mathrm{~cm}^{-2}$ which, adopting $A_{\mathrm{V}}=10\left(A_{\mathrm{K}}=1.1\right.$, Rieke \& Lebofsky 1985) give unreddeded fluxes of $(1.2-1.8) \times 10^{-20} \mathrm{~W} \mathrm{~cm}^{-2}$, about 2-3 times larger than the combined value we obtain for the MIR components $\mathrm{A}$ and $\mathrm{C}$ using the associated radio free-free emission.

The emission coefficient of the $\mathrm{Br} \gamma$ line for $T_{\mathrm{e}}=10^{4} \mathrm{~K}$ is $\epsilon_{\mathrm{Br} \gamma}=5.98 \times 10^{-27} \mathrm{erg} \mathrm{cm}^{-3} \mathrm{~s}^{-1}$. In the case of component A, with $T_{\mathrm{e}}=10^{4} \mathrm{~K}$ and $n_{\mathrm{e}}=4290 \mathrm{~cm}^{-3}$ (see Table 6), the emission coefficient of the [Ne II] line is $\epsilon_{[\mathrm{NeII}] 12.8=}=$ $8.38 \times 10^{-22} \mathrm{erg} \mathrm{cm}^{-3} \mathrm{~s}^{-1}$, while in the case of component $\mathrm{C}$, with $T_{\mathrm{e}}=10^{4} \mathrm{~K}$ and $n_{\mathrm{e}}=2240 \mathrm{~cm}^{-3}, \epsilon_{[\mathrm{NeII}] 12.8}=8.42 \times$ $10^{-22} \mathrm{erg} \mathrm{cm}^{-3} \mathrm{~s}^{-1}$. Following Eq. (1), we obtain $\mathrm{Ne}^{+} / \mathrm{H}^{+}(\mathrm{A})=$ $[1.5(1.8) \pm 0.4] \times 10^{-4}$ and $\mathrm{Ne}^{+} / \mathrm{H}^{+}(\mathrm{B})=[1.6(1.9) \pm 0.4] \times$ $10^{-4}$, where the values in bracktets are obtained by correcting the [Ne II] fluxes from extinction (Sect. 3.3.5). These abundances give a lower limit to the elemental abundance of $\mathrm{Ne}$ of $(1-2)[\mathrm{Ne} / \mathrm{H}]_{\odot}$.

Measurements of the oxygen abundance towards the optical starburst A where these bright infrared knots are located (see Sect. 3.3.1) based on long-slit optical spectra give values between $\sim 0.5$ and $\sim 0.8$ times the solar oxygen abundance (Johansson 1987; Vacca \& Conti 1992; Kobulnicky et al. 1995). These values have been recomputed using the P-method of Pilyugin (2001) for high metallicity regions and considering $12+\log [\mathrm{O} / \mathrm{H}]_{\odot}=8.69$ (Allende Prieto et al. 2001). Our lower limits for the $\mathrm{Ne} / \mathrm{H}$ abundance and the value estimated by Beck et al. (2001) from similar MIR and radio observations (around twice solar) tend to indicate an abundance ratio of solar or higher. This might be the effect of a metal enrichment in the infrared knots or of an enhancement of neon. More likely, this apparent discrepancy is due to an underestimate of the true solar $\mathrm{Ne}$ abundance, as recently suggested from helioseismological and stellar studies. Indeed, our estimated $\mathrm{Ne} / \mathrm{H}$ abundance is subsolar and hence reconciled with a subsolar $\mathrm{O} / \mathrm{H}$ value if we adopt the latest upward revisions of the solar Ne abundance suggested from helioseismology (Antia \& Basu 2005; Bahcall et al. 2005) and from solar type stars (Drake \& Testa 2005). Moreover, we might be underestimating the $\operatorname{Br} \gamma$ line fluxes associated with the MIR components as suggested by the comparison with direct measurements of $\mathrm{Br} \gamma$.

\section{Discussion}

\subsection{Importance of high spatial resolution observations}

Figure 7 shows a comparison between the fluxes measured by TIMMI2 and ISO for NGC 3256, II Zw 40 and NGC 5253 C2. In the case of NGC 3256 (cf. Sect. 3.1.3), only $30 \%$ of the [Ar III] and [Ne II] fluxes comes from the two galactic nuclei. In the case of NGC $5253 \mathrm{C} 2$, only $20 \%$ of the [Ne II] flux measured by ISO comes from this super-star cluster, while the percentages are larger for the [S IV] $(\sim 50 \%)$ and [Ar III] $(\sim 80 \%)$. In our previous work, where we presented the TIMMI2 spectrum of the IR bright nebula C2 in NGC 5253 
Table 6. Summary of properties of the different infrared regions observed in NGC 3256 II Zw 40, He 2-10 and NGC 5253.

\begin{tabular}{lrrrrrr}
\hline \hline \multicolumn{1}{c}{ Property } & NGC 3256 N & NGC 3256 S & II Zw 40 & He 2-10 A & He 2-10 C & NGC 5253 C2 \\
\hline$D(\mathrm{Mpc})^{a}$ & 37 & 37 & 9.2 & 9.0 & 9.0 & $3.3^{(13)}$ \\
Diameter $(\mathrm{pc})^{b}$ & $316^{(1)}$ & $<179^{(6)}$ & $<22.3^{(7)}$ & $5.8^{(10)}$ & $8.4^{(10)}$ & $1.6^{(13)}$ \\
$n_{\mathrm{e}}\left(\mathrm{cm}^{-3}\right)^{c}$ & $1000^{(2)}$ & - & $>1700^{(8)}$ & $4290^{(10)}$ & $2240^{(10)}$ & $50000^{(13)}$ \\
$Q_{0}\left(10^{52} \mathrm{~s}^{-1}\right)^{d}$ & $60^{(3)}$ & $15^{(3)}$ & $10^{(8)}$ & $2.6^{(11)}$ & $1.7^{(11)}$ & $2^{(13)}$ \\
$Z / Z_{\odot}$ & $1.3^{(4)}$ & $1.3^{(4)}$ & $1 / 3^{(9)}$ & $>1.2^{(12)}$ & $>1.2^{(12)}$ & $1 / 3^{(13)}$ \\
$A_{\mathrm{V}}$ & $5.6^{(14)}$ & $10.2^{(14)}$ & $10^{(15)}$ & $10^{(16)}$ & $10^{(16)}$ & $17 \pm 6^{(17)}$ \\
PAH bands & yes & yes & no & yes & yes & $n$ no $^{(13)}$ \\
$([\mathrm{S} \mathrm{IV}] /[\mathrm{Ne} \text { II }])_{\text {TIMMI2 }}{ }^{e}$ & $<0.05$ & $<0.40$ & $>6.7$ & $<0.10$ & $<0.21$ & $12.9 \pm 4.6^{(13)}$ \\
$([\mathrm{S} \mathrm{IV}] /[\mathrm{Ne} \text { II }])_{\text {ISO }}{ }^{\prime}$ & $0.010 \pm 0.002^{(5,18)}$ & $12 \pm 2^{(5)}$ & - & - & $5.6 \pm 0.8^{(5)}$ \\
\hline
\end{tabular}

${ }^{a}$ Distance to the source. ${ }^{b}$ Source diameter. ${ }^{c}$ Electron density. ${ }^{d}$ Ionising photon luminosity. ${ }^{e}$ [S IV]/[Ne II] line ratio observed with TIMMI2. ${ }^{f}[\mathrm{~S} \mathrm{IV}] /[\mathrm{Ne}$ II $]$ line ratio observed with ISO. ${ }^{1}$ Siebenmorgen et al. (2004) has recently obtained an image at $10.4 \mu \mathrm{m}$ with a resolved $(F W H M)$ diameter of 1 .'76. ${ }^{2}$ Estimated from the [S II] $\lambda \lambda 6717 / 6731$ line ratio of 0.96 measured by Lípari et al. $(2000) .{ }^{3}$ Estimated from the extinction-corrected Br $\gamma$ line flux obtained by Kotilainen et al. (1996), see Sect. 3.1.6. ${ }^{4}$ From Lípari et al. $(2000)$ assuming $12+\log (\mathrm{O} / \mathrm{H})_{\odot}=8.69($ Allende Prieto et al. 2001). ${ }^{5}$ From Verma et al. (2003). ${ }^{6}$ Upper limit given by our spectroscopic observations (cf. Sect. 3.1.2). ${ }^{7}$ From the $N$-band image by Beck et al. (2002). ${ }^{8}$ Estimated from the $2 \mathrm{~cm}$ observations by Beck et al. (2002) assuming $T_{\mathrm{e}}=12500 \mathrm{~K}$, see Sect. 3.2.5. ${ }^{9}$ Average value of different estimates (Vacca \& Conti 1992; Pagel et al. 1992; Walsh \& Roy 1993; Masegosa et al. 1994; Guseva et al. 2000; Pérez-Montero \& Díaz 2003). It assumes $12+\log (\mathrm{O} / \mathrm{H})_{\odot}=8.69$ (Allende Prieto et al. 2001). ${ }^{10}$ From Vacca et al. (2002). ${ }^{11}$ Estimate given by Johnson \& Kobulnicky (2003). ${ }^{12}$ See Sect. 3.3.6. ${ }^{13}$ From Martín-Hernández et al. (2005). ${ }^{14}$ See Sect. 3.1.5. ${ }^{15}$ Sect. 3.2.4. ${ }^{16}$ See Sect. 3.3.5. ${ }^{17}$ From Martín-Hernández et al. (2005). ${ }^{18}$ The ISO aperture includes both $\mathrm{N}$ and $\mathrm{S}$ nuclei.

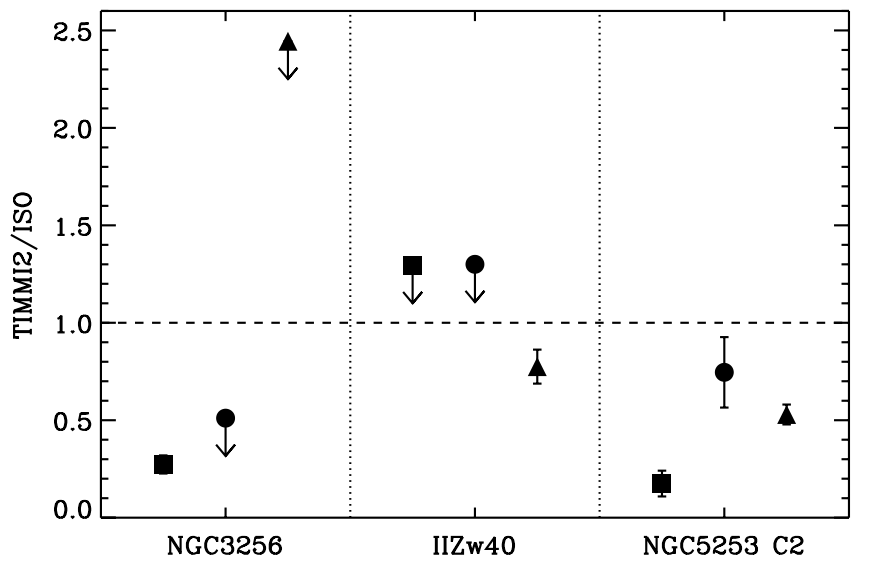

Fig. 7. Comparison between the line fluxes measured by TIMMI 2 and ISO. Symbols correspond to [Ne II] $12.8 \mu \mathrm{m}$ (square), [Ar III] $9.0 \mu \mathrm{m}$ (circle) and [S IV] $10.5 \mu \mathrm{m}$ (triangle). The lines are ordered by increasing ionisation potential: $21.6,27.6$ and $34.8 \mathrm{eV}$ respectively. In the case of NGC 3256, we have added the contributions of the $\mathrm{N}$ and $\mathrm{S}$ nuclei to the TIMMI2 flux since the ISO aperture includes both sources. The dashed line represents the one-to-one relation.

(cf. Martín-Hernández et al. 2005), we showed the implications that this has for the interpretation of line fluxes in terms of the properties (age, IMF, etc.) of the ionising cluster. Only in the case of II Zw 40, there seems to be a good correspondence between ISO and our high spatial resolution observations, suggesting that most (if not all) of the MIR flux of the galaxy is confined to the compact infrared source.

\subsection{A comparison with the infrared supernebula C2 in NGC 5253}

In Table 6 we show a summary of the properties (size, electron density, $Q_{0}$, metallicity, presence of PAHs and excitation) of the sources described in this work. They are compared with the properties of the embedded super-star cluster C2 in NGC 5253 (Martín-Hernández et al. 2005).
Overall the properties compiled in Table 6 are suggestive of the regions/"nuclei" of NGC 3256 as complex regions, possibly hosting several separate star forming regions. Possibly this is also the case for the remaining objects, as it seems quite improbable that regions as extreme as C2 of NGC 5253 are sufficiently common. Indeed, there are already indications, mostly from radio observations, that II Zw 40 and regions A and B of He 2-10 consist of several smaller star forming regions, or superstar complexes (e.g. Beck et al. 2002; Johnson 2005), on spatial scales smaller than the ones achieved here.

Hence, the two nuclei in NGC 3256 are characterised by their large sizes and values of $Q_{0}$, which indicate that they may probably be ionised by multiple clusters having $6 \times 10^{4}$ and $1.5 \times$ $10^{4}$ O7 equivalent stars, respectively. Their MIR spectra show PAHs and their excitation (measured by the $[\mathrm{S} \mathrm{IV}] /[\mathrm{Ne}$ II] line ratio) is relatively low.

The comparison between the compact objects in II Zw 40 and $\mathrm{He} 2-10$ with the embedded supernebula C2 in NGC 5253 is more interesting. The optically thick thermal radio emission and compactness of these sources have lead us to assume them to be "scaled-up" ultracompact H II regions excited by super-star clusters which have recently formed (e.g. Vacca et al. 2002).

The spectrum of $\mathrm{C} 2$ is characterised by a strong [S IV] line and does not show PAHs. On the contrary, the spectra of the MIR components A and $\mathrm{C}$ in $\mathrm{He} 2-10$ show a strong [Ne II] line and have PAHs. The difference between their spectra could be due to the different hardness of the radiation field, as traced by the $[\mathrm{S} \mathrm{IV}] /[\mathrm{Ne}$ II] line ratio and which is directly influenced by the metallicity, among other factors. While the IR sources in He 2-10 might have a solar (or supersolar) metallicity and have a low [S IV]/[Ne II] line ratio, the opposite is true for C2. Even more, it is quite likely that cluster C2 in NGC 5253 is quite different from regions $\mathrm{A}$ or $\mathrm{C}$ in $\mathrm{He} 2-10$ in the sense that it may be much more embedded: it seems that this cluster has no optical counterpart (e.g. Alonso-Herrero et al. 2004). Hence, it is not surprising that their IR spectrum is different.

In terms of metal content and excitation, $\mathrm{C} 2$ is more similar to the compact IR nebula in II Zw 40. However, the nebula in II Zw 40 is not so deeply embedded (visual extinction is of the order of 2-3 mag, although some authors give values as large as 10 mag; see Sect. 3.2.4) and is much less compact than C2. 


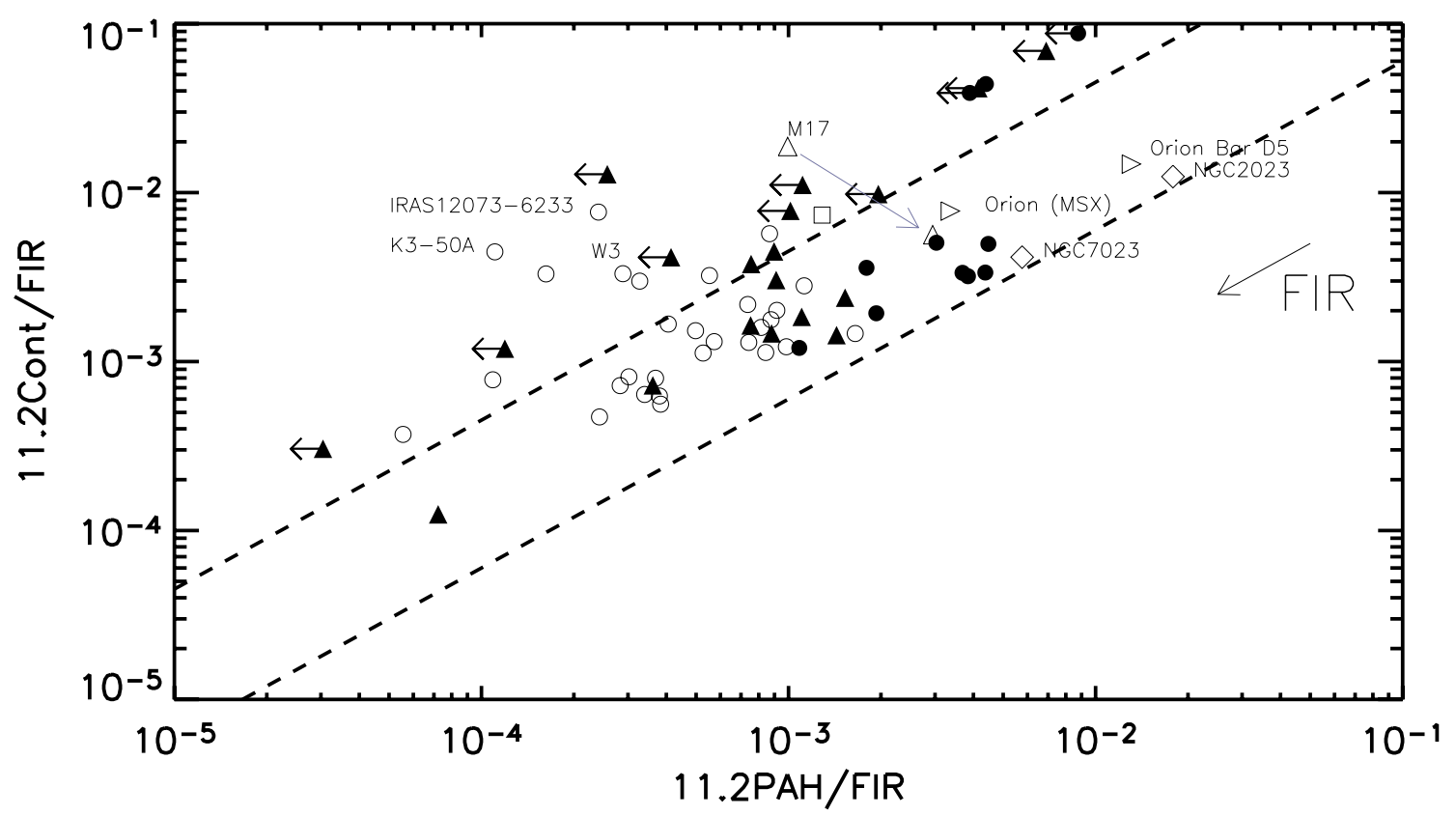

Fig. 8. Ground-based MIR/FIR diagnostic diagram for star forming regions. Galactic H II regions are presented by open circles, Orion by right triangles, the extended H II region M 17 by open triangles, reflection nebulae (RNe) by open diamonds, 30 Doradus by an open square, TIMMI2 observations of extra-galactic nuclei and star clusters by filled triangles (this work and Siebenmorgen et al. 2004) and ISOPHOT observations of extra-galactic nuclei by filled circles (Siebenmorgen et al. 2004). The dotted lines correspond to a $11.2 \mathrm{PAH} / \mathrm{continuum}$ ratio of 0.22 and 1.66 from top to bottom. The effect of an overestimate of the far-IR (FIR) flux by a factor of 2 is indicated by an arrow parallel to the dotted lines. The second arrow indicates the shift in position from the pointing inside the H II region towards that of the molecular cloud in the extended star forming region M 17. This diagram is being adapted from that of Peeters et al. (2004b) using the $11.2 \mu \mathrm{m}$ PAH instead of the $6.2 \mu \mathrm{m}$ PAH.

\subsection{PAH emission}

\subsubsection{The ground-based MIR/FIR diagnostic diagram for star forming regions}

Peeters et al. (2004b) have examined the use of PAH bands as tracers of star formation. In order to distinguish the different natures of the galaxies, i.e. AGN-dominated, starburst-dominated or heavily obscured, these authors present a new MIR/FIR diagnostic, along the lines of previous works by e.g. Genzel et al. (1998), Laurent et al. (2000) and Clavel et al. (2000), based on the ratio of the $6.2 \mu \mathrm{m}$ PAH emission band to FIR flux and the ratio of the $6.2 \mu \mathrm{m}$ continuum to FIR flux. These two ratios isolate the strongly obscured galaxies while the 6.2 PAH-to-continuum ratio provides a clear measurement of any AGN contribution to the MIR. The comparison with Galactic objects reveals that most of the Ultra-Luminous IR Galaxies (ULIRGs) are found co-located with compact $\mathrm{H}$ II regions in contrast to normal and starburst galaxies, which are mainly co-located with exposed PDRs.

A problem with this and other diagrams is that they are not accessible to ground-based observations. We have no access to the 6.2 or $7.7 \mu \mathrm{m}$ PAH bands through the atmospheric MIR window $(\sim 8-13 \mu \mathrm{m})$ and the same difficulty arises in the cases of the diagnostics by Genzel et al. (1998) and Laurent et al. (2000), which are also based on lines such as [O IV] at $25.9 \mu \mathrm{m}$ or the ratio of warm $(14-15 \mu \mathrm{m})$ to hot $(5.1-6.8 \mu \mathrm{m})$ dust continuum. Therefore, we have adapted the MIR/FIR diagnostic diagram presented in Peeters et al. (2004b) in order to include the $11.2 \mu \mathrm{m}$ PAH band and the $11.2 \mu \mathrm{m}$ continuum instead and test whether similar conclusions are reached compared to previous works based upon the 6.2 or $7.7 \mu \mathrm{m}$ PAH bands.

The use of the $11.2 \mu \mathrm{m}$ PAH band has however clear consequences on the interpretation. The PAH emission band and the dust continuum at this wavelength are likely to trace different carriers due to a difference in temperatures, size, heating mechanism and charge state in the case of the PAHs. To first order, the fraction of total PAH flux emitted in the $11.2 \mu \mathrm{m}$ PAH band varies only from 9 to $27 \%$ with an average of $18 \pm 5 \%$ and that emitted in the $6.2 \mu \mathrm{m}$ PAH band varies only from 14 to $38 \%$ with an average of $28 \pm 4 \%$ (Peeters et al. 2002a). Given the order of variation observed in the $6.2 \mathrm{cont} / \mathrm{FIR}$ (Peeters et al. 2004b) and 11.2 cont/FIR (see Fig. 8), both ratios are representative of the PAH/FIR ratio. However, this clearly does not hold for the dust continuum emission.

The 11.2 MIR/FIR diagnostic diagram is shown in Fig. 8. Note that the FIR fluxes of the extra-galactic sources have been calculated from the IRAS fluxes and except for NGC $3256 \mathrm{~N}$ and $\mathrm{S}$, no correction has been made to account for the aperture difference. As a consequence, the FIR fluxes presented here are likely overestimated and the use of an "aperture corrected" FIR flux will move the sources in the opposite direction to that indicated by the FIR arrow shown on the plot. However, since most of the observed extra-galactic nuclei and star clusters are probably the dominating source of the FIR flux, this correction is expected to have little influence on the results we discuss here.

The sample of Galactic sources shows a general correlation of the strength of the 11.2 PAH/FIR with the $11.2 \mathrm{cont} / \mathrm{FIR}$. This is very similar to that seen for the MIR/FIR diagram based upon the $6.2 \mu \mathrm{m}$ band, as discussed by Peeters et al. (2004b). This general correlation reveals that the 11.2 PAH-to-continuum ratio is relatively constant in the full sample while the 11.2 PAH/FIR ratio changes by almost three orders of magnitude. Typically, the 11.2 PAH-to-continuum ratio is very low for deeply embedded ultra-compact $\mathrm{H}$ II regions (like e.g. W3, K3-50A) and increases when the characteristics of the region changes to that of an exposed PDR such as Orion and M 17. 
As shown by Fig. 8, the galaxies observed with ISOPHOT by Siebenmorgen et al. (2004), represented by filled circles, are located close to the exposed PDRs such as Orion and M 17. A similar result was found by Peeters et al. (2004b) for ISOPHOT observations of normal and starburst galaxies in the 6.2 MIR/FIR diagram. In contrast, galaxies observed with TIMMI2 (filled triangles) are found to have a lower 11.2 PAH/FIR ratio and thus to be located nearer to the Galactic H II regions. For the 6.2 MIR/FIR diagram, this is also observed for the ULIRGs (Peeters et al. 2004b). This difference between the ISOPHOT and TIMMI2 observations is likely due to the aperture difference between the ISOPHOT, TIMMI2 and IRAS data (the latter for the FIR determination). The much larger ISOPHOT aperture $\left(\sim 20^{\prime \prime}\right)$ probably includes much of the surrounding PDRs while the TIMMI2 slit measures mainly the central emission of the HII region or nucleus although it does cross the ionisation front and the PDR where the PAH emission originates from (e.g. Sellgren et al. 1990; Tielens 1993; Tielens et al. 1993; Verstraete et al. 1996). This is similar to the results obtained by Siebenmorgen et al. (2004) that compare the ISOPHOT data and TIMMI2 spectra of galaxies. In the 6.2 MIR/FIR diagram, the ISOPHOT observations of normal galaxies and starburst galaxies are also found close to the location of exposed PDRs and are not co-located with the Galactic H II regions (Peeters et al. 2004b). This suggests that the PAH emission is probably diffuse and extended and thus difficult to detect by TIMMI2. Consequently, the PAH emission rather seems to trace exposed PDRs and the ISM of galaxies (as seen by ISOPHOT) than the dense star forming regions (as seen by TIMMI2).

A large number of our sources show no evidence of PAH emission. The extra-galactic sources where PAHs are detected are situated between the two dotted lines in Fig. 8 while those without PAH detections are found above the top dotted line, indicating that in these objects the 11.2 PAH-to-continuum ratio is lower. This is not influenced by an overestimate of the FIR flux since a lower FIR flux would move the sources in the opposite direction to the FIR arrow, parallel to the dotted lines; neither it is influenced by the fact that we are dealing with upper limits. In fact, if the upper limit becomes more stringent, the source will move horizontally towards the left, giving an even lower 11.2 PAH-to-continuum ratio. Together with these extragalactic sources, some Galactic H II regions also fall above these dotted lines, all of which are ultra-compact $\mathrm{H}$ II regions which have very strong radiation fields and MIR dust continuum.

Thus, we have constructed a new MIR/FIR diagnostic diagram based on the $11.2 \mu \mathrm{m}$ PAH band and the $11.2 \mu \mathrm{m}$ continuumm and proven that the same conclusions are reached when compared to previous works based upon the 6.2 or $7.7 \mu \mathrm{m}$ PAH bands. This is useful since the $11.2 \mu \mathrm{m}$ PAH band is easily accessible from ground-based observations.

\subsubsection{The $[\mathrm{SIV}] /[\mathrm{NeII}]$ line ratio and the survival of PAHs}

We now analyse the effect of the hardness of the radiation field, i.e. the survival of PAHs in environments with energetic photons. We have a measure of the hardness of the radiation field through the $[\mathrm{S} \mathrm{IV}] /[\mathrm{Ne}$ II] line ratio. Assuming that all sources have the same $\mathrm{S} / \mathrm{Ne}$ abundance ratio, variations in $[\mathrm{S} \mathrm{IV}] /[\mathrm{Ne}$ II $]$ probe the hardness of the radiation field at energies above $\sim 22 \mathrm{eV}$. Figure 9 compares $[\mathrm{SIV}] /[\mathrm{Ne}$ II] with the 11.2 PAH-to-continuum ratio for the objects analysed in this work (cf. Table 6) and those observed by Siebenmorgen et al. (2004) also using TIMMI2. This figure clearly shows that sources with PAHs have a [S IV] $/[\mathrm{Ne}$ II] ratio $\lesssim 0.35$, while sources that do not show PAHs

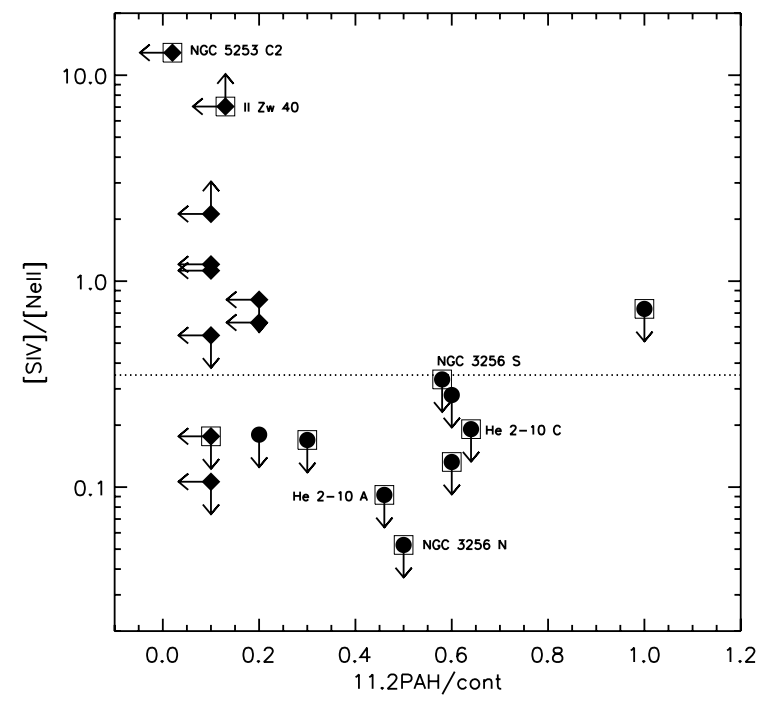

Fig. 9. Comparison of the [S IV] $10.5 \mu \mathrm{m} /[\mathrm{Ne}$ II $] 12.8 \mu \mathrm{m}$ line ratio with the $11.2 \mu \mathrm{m} \mathrm{PAH/continuum} \mathrm{ratio} \mathrm{for} \mathrm{extra-galactic} \mathrm{nuclei} \mathrm{and}$ embedded super-star clusters. We combine the sources in Table 6 with the galactic nuclei observed by Siebenmorgen et al. (2004) using also TIMMI2. Sources plotted as circles show PAH bands in their MIR spectra and are concentrated predominantly below [S IV $] /[\mathrm{Ne}$ II] $\sim 0.35$. Sources plotted as diamonds do not show PAHs and have mostly line ratios above this limit of 0.35 . Nuclei/star clusters in starburst galaxies are marked by a square. The other sources are nuclei in Seyfert galaxies.

predominantly have $[\mathrm{S}$ IV $] /[\mathrm{Ne}$ II] ratios above 0.35 . Exceptions to this behaviour are Centaurus $\mathrm{A}$ and $\mathrm{M} \mathrm{83,} \mathrm{which} \mathrm{do} \mathrm{not}$ show PAHs but still have a [S IV]/[Ne II] ratio lower than 0.35 . Centaurus A is the closest example of an AGN. While the spectrum of this galaxy obtained through large $\left(>20^{\prime \prime}\right)$ apertures is PAH dominated, the 3 " spectrum by Siebenmorgen et al. (2004) is featureless except for the presence of a strong [ $\mathrm{Ne} \mathrm{II]} \mathrm{line} \mathrm{and}$ is very similar to the nucleus of M 83 .

Starburst galaxies are very similar to Galactic H II regions, thus we have checked this relationship between the hardness of the radiation field and the presence of PAHs using a large sample of Galactic H II regions observed with ISO/SWS (Peeters et al. 2002b). While all the HII regions in the catalogue show the presence of PAHs, only 4 of them have a [S IV]/[Ne II] ratio > 0.35: W3A, IRAS 12063-6259, IRAS 12073-6233 and IRAS 11143-6134. Their ISO/SWS spectra clearly show PAH emission bands. However, we have recently obtained TIMMI2 slit spectra for 2 of these sources, IRAS 12063-6259 and IRAS 12073-6233. For neither of them have we detected PAH emission bands in their long-slit spectra (Martín-Hernández et al., in preparation), even though the TIMMI2 slit did cross the ionisation front and hence the PDR where the PAH emission originates from. As mentioned above, this suggests that the PAH emission is probably diffuse and extended and hence not dominated by the denser regions but rather by the exposed PDR and ISM. This diffuse emission might be difficult to detect by TIMMI2 due to its narrow slit.

The apparent relation between the hardness of the radiation field and the intensity of the PAH emission is consistent with recent results by Madden et al. (2006). These authors found a strong correlation between the $[\mathrm{Ne}$ III] $/[\mathrm{Ne}$ II $]$ ratio, tracing the hardness of the radiation field and the $\mathrm{PAH} /$ dust intensity ratio for a wide range of objects, including low-metallicity galaxies as well as Galactic H II regions and other metal-rich galaxies. 
They characterise the dust emission as the fitted, feature-free continuum of their MIR spectra between 10 and $16 \mu \mathrm{m}$. These authors conclude that this hard radiation field must play an important role in the destruction of PAHs in low metallicity regions.

The lack and/or weakness of the PAH emission bands in galaxies or $\mathrm{H}$ II regions with a hard radiation field (i.e. $[\mathrm{S} \mathrm{IV}] /[\mathrm{Ne}$ II $]>0.35$ ) may be due to a low PAH abundance as a consequence of e.g. (i) $\mathrm{PAH}$ dehydrogenation, i.e. the $\mathrm{CH}$ bond rupture due to the absorption of a UV photon (since we are using the $11.2 \mu \mathrm{m}$ PAH emission band as a tracer for the presence of PAHs); (ii) PAH destruction; (iii) the relative contribution of the different phases in the ISM or (iv) PAH-dust competition, i.e. PAHs are not being excited due to the presence of dust located inside the H II regions. In principle, a low PAH abundance could also originate from a low PAH formation rate due to a dust formation process that is different to that occuring in our Galaxy as e.g. may be the case in low metallicity galaxies. However, we deem this possibility unlikely to explain the general relation we find between hardness of the radiation field and low PAH fluxes since our sample also includes Galactic H II regions without PAH detections.

Here we discuss the four possibilities mentioned above:

(i) PAH dehydrogenation: we have traced the presence or nonpresence of PAHs in this work through the detection of the $11.2 \mu \mathrm{m}$ PAH band. To first order, the PAH/FIR can be traced by the 11.2 PAH/FIR since (1) dehydrogenation of PAHs (which would effect the strength of this $\mathrm{CH}$ mode) does not seem to be important (see below) and (2) the fraction of total PAH flux emitted in the $11.2 \mu \mathrm{m}$ PAH band varies only from 9 to $27 \%$ with an average of $18 \pm 5 \%$ (Peeters et al. 2002a). Indeed, space-based observations suggest that in regions with low or no PAH emission (as is the case of e.g. II Zw 40) the strength of all PAH emission bands decreases simultaneously and not only that of the $11.2 \mu \mathrm{m}$ PAH band (e.g. Madden 2000; Laurent et al. 2000; Madden et al. 2006). In addition, ISO/SWS observations of a large sample of sources as well as theoretical calculations show that dehydrogenation of PAHs should have no effect on the observed PAH spectrum (for a summary, see Hony et al. 2001).

(ii) $\mathrm{PAH}$ destruction: in order to investigate $\mathrm{PAH}$ destruction as a consequence of the hardness of the radiation field as traced by ratios such as $[\mathrm{S} I V] /[\mathrm{Ne} \mathrm{II}]$, one should keep in mind that PAH emission originates inside the PDR and thus is not colocated with the high energy photons found within H II regions.

The strength of the radiation field at the location where the PAH emission originates, the PDR, is generally expressed by the incident FUV flux between 6 and $13.6 \mathrm{eV}$ measured in units of the average interstellar radiation field $\left(1.6 \times 10^{-6} \mathrm{~W} / \mathrm{m}^{2}\right.$, Habing 1968). This quantity is called $G_{0}$. We have estimated the values of $G_{0}$ for the objects in our sample and those in the study by Siebenmorgen et al. (2004). They have been calculated using the dust temperature, $T_{\mathrm{d}}$, derived from ISO/LWS observations (Negishi et al. 2001) and the relation between $G_{0}$ and $T_{\mathrm{d}}$ given by Hollenbach et al. (1991) with $A_{\mathrm{V}}=0.5$ (Negishi et al. 2001). In the cases where $T_{\mathrm{d}}$ is not known, we have used the relation between the gas density, $n$, and the ratio of the IRAS 60 and $100 \mu \mathrm{m}$ fluxes together with the relation between $G_{0}$ and n (Negishi et al. 2001). In any case, the obtained $G_{0}$ is an average for the entire galaxy due to the large LWS and IRAS apertures. Also note that these two methods ignore metallicity effects and thus the values of $G_{0}$ we obtain are less accurate in the case of low metallicity galaxies. $\log \left(G_{0}\right)$ is found to be in the range of 1.8-3.7 dex with an average value of $3.0 \pm 0.4 \mathrm{dex}$. No correlation is found between $G_{0}$ and the PAH strength or between $G_{0}$ and the presence or non-presence of PAH emission bands in either the TIMMI2 or ISO observations. Furthermore, this range in $G_{0}$ is similar to the values typically seen in Galactic RNe (Young Owl et al. 2002), whose IR spectra are dominated by PAH emission bands. Hence, this suggests that it is unlikely that the low 11.2 PAH-to-continuum ratio reflects a destruction of PAHs in these environments (i.e. PDRs).

The $G_{0}$ values we estimate correspond to the average $G_{0}$ of the PDRs in the galaxy because of the large apertures (LWS, IRAS) we are considering and might not be representative of the conditions in the small/unresolved sources we are studying here. The above conclusion must be then taken with caution. For low metallicity galaxies it is found that the PDRs could be restricted to very small dense clumps (Madden 2000; Galliano et al. 2005). If that is the case, the estimated $G_{0}$ 's would then be an average of such clumps.

(iii) Relative contribution of different regions: rather than invoking a special PAH destruction, the low PAH emission could just be a consequence of the relative contribution of the different phases of the interstellar medium in the beam. For example, a substantial pervasive, diffuse and highly ionised medium is found throughout low metallicity galaxies (Madden et al. 2006) and - as known from Galactic H II regions (e.g. Tielens et al. 1993; Verstraete et al. 1996) - PAHs do not survive inside highly ionised mediums. The presence of a diffuse ionised medium implies a smaller contribution of the diffuse neutral interstellar medium (PDRs, ISM). This neutral medium contributes considerably to the observed PAH emission in starburst and normal galaxies (see above and e.g. Peeters et al. 2004b) and hence, such contribution to the PAH emission would then be missing in low metallicity galaxies and would explain the low PAH abundances in these galaxies. This is consistent with what we said earlier regarding the aperture effect on the detection of PAHs: PAHs are more prominent in large aperture observations because the relative contribution of the PDRs and ISM with respect to the highly ionised medium of the $\mathrm{H}$ II regions is larger.

(iv) $\mathrm{PAH}$-dust competition: another explanation might be found in the competition for FUV photons by the dust grains and the PAH molecules. Dust grains are able to reside inside the H II region (see e.g. M 17, Fig. 3 of Verstraete et al. 1996). When this occurs, the dust located inside the HII region absorbs a large fraction of photons before they reach the PDR. In particular, the optical depth of dust in H II regions, $\tau$ (dust), is proportional to $n^{1 / 3}$. Hence, at high densities, more of the FUV photons are absorbed by dust in the HII region than at low densities. Moreover, a larger fraction of the ionising photons is absorbed by the dust rather than the ionised gas. As a result, PAHs, which are located outside of the H II regions, are less excited. In addition, the dust inside the $\mathrm{H}$ II region attains very high temperatures and consequently the PAH-to-continuum ratio is lower. Indeed, Fig. 8 shows a relatively large spread in the 11.2 PAH-to-continuum ratio with the lowest ratios corresponding to the highly excited sources. This is also nicely illustrated by the two different ISO pointings towards the extended H II region M 17 plotted in Fig. 8, one inside the $\mathrm{HII}$ region and the other towards the molecular cloud. Thus, the absence of PAH emission in highly excited sources might be due to a lower 
availability of exciting photons due to the presence of dust within the H II region.

Thus, the relation between the hardness of the radiation field and the presence or non-presence of PAHs does not necessarily imply PAH destruction. Since PAHs originate inside the PDR and thus are not co-located with the high energy photons found within H II regions, this relation might be connected to the presence of dust within the $\mathrm{H}$ II region leading to a PAH-dust competition for UV photons. We consider also the scenario where the low PAH emission is a consequence of the relative contribution of the different phases of the interstellar medium, in particular, the presence of a pervasive and highly ionised medium.

\subsection{The stellar content of the IR supernebula in II Zw 40}

It has been shown in Sect. 3.2 that most (if not all) of the MIR line fluxes of II Zw 40 measured by ISO is confined to the compact (0.'5) source observed by TIMMI2. We can use then the large number of MIR lines observed by ISO to constrain properties of the ionising cluster following the analysis done with NGC 5253 C2 in our previous work (Martín-Hernández et al. 2005).

Stellar properties can be derived from nebular lines using photoionisation models. These photoionisation models depend on the SED of the ionising cluster (age and IMF), the local abundance and the ionisation parameter, $U$. In particular, the parameter $U$, which depends on the geometry, is usually difficult to constrain.

We compute sets of nebular models with the photoionisation code CLOUDY ${ }^{2}$ (Ferland et al. 1998) version 96.00 using $\mathrm{MICE}^{3}$, the IDL interface for CLOUDY created by H. Spoon. The computation is performed for a static, spherically symmetric, ionisation bounded gas distribution with an inner cavity. We assume that the gas is uniformly distributed in small clumps of constant density over the nebular volume and occupies a fraction $\epsilon$ of the total volume. The input parameters of the photoionisation models are: the shape of the SED, the ionising photon luminosity $\left(Q_{0}\right)$, the electron density $\left(n_{\mathrm{e}}\right)$, the inner radius of the shell of ionised gas $\left(R_{\text {in }}\right)$, the filling factor $(\epsilon)$, and the chemical composition $(Z)$.

We have used the evolutionary synthesis code Starburst $99^{4}$ (Leitherer et al. 1999) version 4.0 to model the integrated properties of the stellar cluster. We assume an instantaneous burst of star formation, a Salpeter (1955) IMF with exponent $\alpha=2.35$ $\left(\mathrm{d} N / \mathrm{d} \ln m \propto m^{1-\alpha}\right)$, a lower mass cutoff $M_{\text {low }}=1 M_{\odot}$ and an upper mass cutoff $M_{\text {up }}$ set to 30,50 and $100 M_{\odot}$. We also assume that the stars evolve from the main sequence following the $Z=0.008$ stellar tracks. Stellar tracks with $Z=0.006$, the metallicity measured for II Zw 40, are not available but the effect of decreasing $Z$ will be discussed below. We present models every $0.5 \mathrm{Myr}$ for $10 \mathrm{Myr}$ after the burst of star formation.

The chemical composition of the gas is set to $Z=0.006$ (cf. Table 6). We have adjusted the helium abundance $Y$ according to $Y=Y_{\mathrm{p}}+(\Delta Y / \Delta Z) Z$, where $Y_{\mathrm{p}}=0.24$ is the primordial helium abundance (Audouze 1987) and $\Delta Y / \Delta Z=3$ is an observed constant (Pagel 1992). To arrive at the appropriate metal abundances, we have simply scaled the solar values stored in the CLOUDY database.

A change of the rate of Lyman ionising photons, electron density, inner radius of the shell and/or filling factor is

\footnotetext{
2 see http://wWw. nublado.org

3 see http://isc. astro. cornell. edu/ spoon/mice.html

4 see http://wWw. stsci.edu/science/starburst99/
}

equivalent to a change of the ionisation parameter $(U)$ defined by CLOUDY as:

$$
U=Q_{0} /\left(4 \pi R_{\mathrm{in}}^{2} n_{\mathrm{e}} c\right) .
$$

There exists a relationship between $Q_{0}, n_{\mathrm{e}}$, the inner radius $R_{\text {in }}$, the outer radius $R_{\text {out }}$ and the filling factor $\epsilon$ given by Martín-Hernández et al. (2005):

$\left(1-x^{3}\right) R_{\mathrm{out}}^{3} \epsilon=\frac{3 Q_{0}}{4 \pi n_{\mathrm{e}}^{2} \alpha_{\mathrm{B}}}$

where $R_{\text {in }}=x R_{\text {out }}$ and $0<x<1$. Physical solutions of this equation are those that satisfy the condition $\epsilon \leq 1$.

Considering $Q_{0}=10^{53} \mathrm{~s}^{-1}$, an electron density of $\sim 1700 \mathrm{~cm}^{-3}$ and an outer radius $R_{\text {out }}=11.15 \mathrm{pc}$ (cf. Table. 6 ), together with the above condition, we obtain that $R_{\text {in }} \leq 0.38 \times$ $R_{\text {out }}=4.3$ pc, which gives a value of $\log U \geq-0.05$ (cf. Eq. (2)). More compact geometries (i.e. with smaller $R_{\text {out }}$ and bigger densities) will have larger values of the ionisation parameter. We will consider then a nebula with the following parameters: $Q_{0}=10^{53} \mathrm{~s}^{-1}, R_{\text {in }}=4.3 \mathrm{pc}, n_{\mathrm{e}}=1700 \mathrm{~cm}^{-3}$ and $\epsilon=1$. The effect of a larger ionisation factor will be discussed.

Figure 10 shows the results of the photoionisation models as a function of time since the burst of star formation. The same conclusions as in the case of the super-star cluster C2 in NGC 5253 can be reached. I.e. we find two possible solutions for the age and upper mass cutoff of II Zw 40: 1) a young ( $\$ 3-4 \mathrm{Myr}$ ) cluster with a low upper mass cutoff $M_{\text {up }}<50 M_{\odot}$; and 2) a cluster of $\sim 4-7$ Myr with a standard high upper mass cutoff $M_{\text {up }} \sim 100 M_{\odot}$.

All line ratio predictions shown in Fig. 10, except [O IV]/Br $\gamma$, are compatible with this result. In the case of $[\mathrm{ArIII}] / \mathrm{Br} \gamma$, $[\mathrm{S} \mathrm{III}] / \mathrm{Br} \gamma$, and $[\mathrm{Ne} \mathrm{II}] / \mathrm{Br} \gamma$, these line ratios are not very sensitive or their uncertainties are too large.

The [O IV] line is known to be difficult to reproduce by pure stellar photoionisation (Lutz et al. 1998; Schaerer \& Stasińska 1999; Martín-Hernández et al. 2005), but could also be related to non-stellar processes (cf. Lutz et al. 1998; Viegas et al. 1999), as indicated e.g. by recent IRS/Spitzer observations of NGC 5253 by Beirao et al. (2006). This underprediction of the [O IV] line flux by the models was also encountered in the case of NGC 5253 C2, where it was concluded that a modification of the adopted SEDs close to and beyond the He II edge (note that the ionisation edge for the creation of O IV, $54.9 \mathrm{eV}$, is the hightest of all the considered ions) was necessary in order to reproduce all the MIR lines.

The increase of the ionisation parameter, whose effect on the predictions is indicated by arrows in Fig. 10, will only accentuate the differences between the observations and the models with $M_{\text {up }}=100 M_{\odot}$. Consequently, if the nebula had a higher parameter $U$, the upper mass cutoff of the IMF would need to be revised downward in comparison with the models discussed here. The same holds in the case of using SEDs with a lower metallicity or adding internal dust in the nebula. These and other factors such as varying the power law index of the IMF and the density law or considering a matter bounded geometry for the nebula are discussed in detailed in Martín-Hernández et al. (2005). There it was shown that any of these effects can reconcile the models with $M_{\text {up }}=100 M_{\odot}$ with the observations. An age <3-4 Myr would agree with the optically thick thermal bremsstrahlung origin of the radio emission associated with the IR knot and the lack of supernova signatures (cf. Sect. 3.3.1). However, it would imply an IMF with a low upper mass cutoff $\left(M_{\text {up }} \lesssim 50 M_{\odot}\right)$. The solution of an older cluster of $\sim 4-7$ Myr would imply that it is 

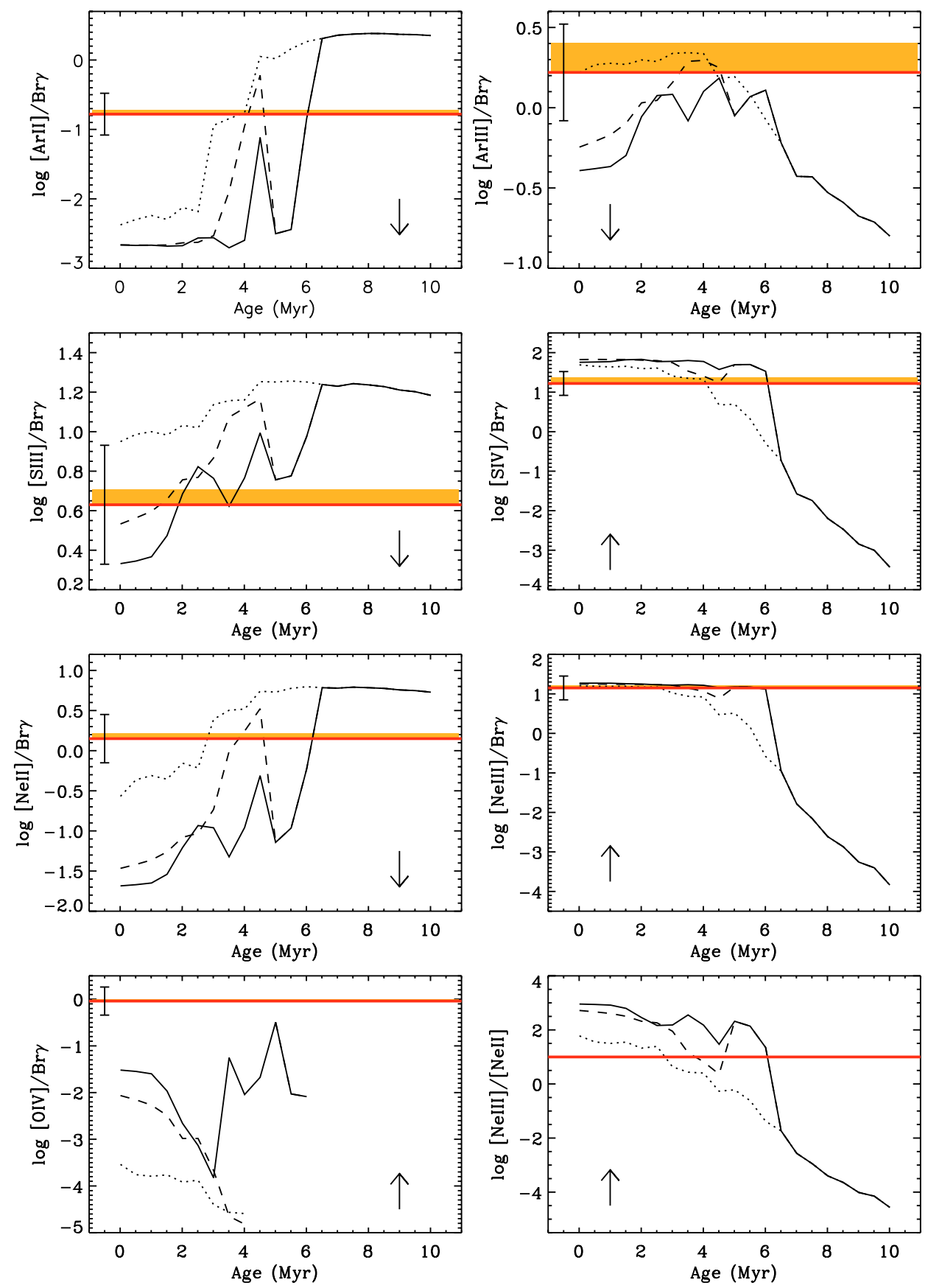

Fig. 10. Variation of selected emission line ratios as a function of starburst age. The observed line ratios are indicated by the horizontal line. The [Ar III] 9.0, [S IV] 10.5 and [Ne II] $12.8 \mu \mathrm{m}$ lines were measured with TIMMI2 (cf. Table 3). The lines of [Ar III] 7.0, [Ne III] 15.5 and [S III] 18.7 $\mu \mathrm{m}$ were measured with ISO (Verma et al. 2003). Br $\gamma$ is estimated from the $2 \mathrm{~cm}$ free-free emission (see Sect. 3.2.5) and we show as an error bar on the left side of each panel the effect of having under/overestimating this line flux by a factor of 2 . Plots involving ratios with lines measured within the same aperture such as [S IV]/[Ne II] and [S IV]/[Ar III] give the same result as that of [Ne III]/[Ne II] vs. age. The light band shows the effect of correcting the MIR lines for a visual extinction of $10 \mathrm{mag}$ (cf. Sect. 3.2.4). Solid lines correspond to models with $M_{\text {up }}=100 M_{\odot}$, dashed lines to models with $M_{\text {up }}=50 M_{\odot}$ and dotted lines to models with $M_{\text {up }}=30 M_{\odot}$. The nebular parameters of these models are $Q_{0}=10^{53} \mathrm{~s}^{-1}, R_{\text {in }}=4.3 \mathrm{pc}$, $R_{\text {out }} \sim 11 \mathrm{pc}, n_{\mathrm{e}}=1700 \mathrm{~cm}^{-3}$ and $\epsilon=1$, which correspond to $\log U \sim-0.05$. The effect of increasing the parameter $\mathrm{U}$ and/or decreasing the metallicity of the stellar cluster and/or adopting a steeper IMF and/or including internal dust is indicated by arrows.

possible to contain such compact regions for a longer time that what it is generally though while at the same time, hide the signatures of supernovae. The bright compact nebula in II Zw 40 might thus be the second object, together with NGC $5253 \mathrm{C} 2$, for which indications of a "non-standard" IMF with a low upper mass cutoff $M_{\text {up }} \lesssim 50 M_{\odot}$ exist. Whether this is a characteristic common in compact objects or whether this is due to defects in the stellar and/or nebular modelling remains to be clarified.
Generally fairly young ages are found from stellar populations studies in the optical (e.g. Raimann et al. 2000; Kong et al. 2003; Westera et al. 2004). In particular WR features indicate ages of 3-5 Myr (cf. Schaerer \& Stasińska 1999; Guseva et al. 2000). However, it is not clear if and how this population relates to the one responsible for the mid-IR line emission.

A solution that might lead to the lowering of the ionisation parameter and thus to the reconciliation of model and 
observations is the presence of multiple clusters within the compact core we are considering here. Indeed, the high spatial resolution observations at $2 \mathrm{~cm}$ presented by Beck et al. (2002) reveal 3 or 4 peaks within the central radio emission. They argue that these knots may simply be peaks of the more extended distribution, but also consider the possibility of young H II regions with diameters of the order of $\sim 1$ pc. Förster Schreiber et al. (2001) show that regions with multiple H II regions are best modelled by a randomised distribution of clouds and clusters described by an effective ionisation parameter $U_{\text {eff }}$. The computation of $U_{\text {eff }}$ depends, however, on the detailed knowledge of the properties and distribution of the ionising stars and gas clouds. For the well known starburst galaxy M 82, Förster Schreiber et al. (2001) obtain a value of the effective ionisation parameter lower than using the conventional definition (cf. Eq. (2)) due to the important increase in surface area of the gas exposed to the Lyman continuum radiation field for the more realistic randomised distribution. Indeed such a general tendency can be expected for multiple independent $\mathrm{H}$ II regions producing the same Lyman continuum flux as a single region. However, the opposite trend may be the case in sufficiently compact environments once the multiple H II regions start to overlap, or if they provide a non negligible external ionising radiation field (e.g. due to some leakage). In this case the outer parts, host of emission from low ionisation species, can be eroded or destroyed, leading hence to a global shift toward emission of higher ionisation. Which situation applies to II Zw 40 cannot be judged from the currently available observations.

\section{Conclusions}

We have presented $N$-band spectra $(8-13 \mu \mathrm{m})$ of some locations in three starburst galaxies. In particular, the two galactic nuclei of the spiral galaxy NGC 3256 (North and South), the compact infrared supernebula in the dwarf galaxy II Zw 40 and the two brightest infrared knots in the central starburst of the WR galaxy He 2-10 (named A and C). These spectra have been obtained with TIMMI 2 on the ESO $3.6 \mathrm{~m}$ telescope.

The spectra show an ample variety in terms of continuum, lines and molecular band strength. The two nuclei of NGC 3256 and the two IR knots in $\mathrm{He} 2-10$ show a rising dust continuum, $\mathrm{PAH}$ bands and a strong [Ne II] line. On the contrary, the infrared knot in II Zw 40 is characterised by a rather flat continuum, a strong [SIV] line and does not show the presence of PAH bands.

We demonstrated the value of this type of data in constraining properties such as the extinction in the MIR and metallicity. We found: (1) There is an indication of supersolar metallicity for the two nuclei of NGC 3256, while a possible enhancement of neon with respect to oxygen is found in the two IR knots observed in He 2-10. However, if the latest upward revision of the solar Ne abundance by $\sim 0.4-0.5$ dex suggested from helioseismology and from solar type stars is confirmed, our $\mathrm{Ne}$ abundance estimates could be solar or even (slightly) sub-solar. (2) Around $60 \%$ of the sulphur in the bright IR knot in II Zw 40 could be in the form of $\mathrm{S}^{4+}$, indicating the presence of photons in excess of $47 \mathrm{eV}$. We note however that these abundance estimates largely depend on the assumed flux for the $\operatorname{Br} \gamma$ line. Hence, they must be taken with caution since the MIR and Bry line fluxes are not measured on the same aperture.

We have shown the importance of high spatial resolution observations of extra-galactic sources when compared to observations obtained with larger apertures such as ISO. Only such observations provide accurate measurements of the line and molecular band fluxes emitted by the cluster or galactic nucleus.

We adapted the MIR/FIR diagnostics of Peeters et al. (2004b) in order to use the $11.2 \mu \mathrm{m}$ PAH band, accessible to ground-based observations, instead of the $6.2 \mu \mathrm{m}$ one and compared our observations and those of Siebenmorgen et al. (2004) with Galactic H II Regions. We find that the extra-galactic nuclei and star clusters observed at high spatial resolution (as is the case of the TIMMI2 observations) are closer in this diagram to compact H II regions, while galaxies observed by large apertures such as ISO are nearer to exposed PDRs such as Orion. This is likely due to the aperture difference, where the much larger ISO aperture probably includes much of the surrounding PDRs while the TIMMI2 slit measures the central emission of the H II region.

We have also found a dependence between the PAH presence and the hardness of the radiation field, as measured by the $[\mathrm{SIV}] /[\mathrm{Ne} \mathrm{II}]$ ratio: sources with $\mathrm{PAH}$ emission have a $[\mathrm{S} \mathrm{IV}] /[\mathrm{Ne}$ II] ratio $\lesssim 0.35$, while sources that do not show PAHs have line ratios above 0.35 . We investigated possible origins for this relation and conclude that it does not necessarily imply PAH destruction, but could also be explained by the PAH-dust competition for FUV photons. Dust grains probably absorb a large fraction of the UV photons before they reach the PDR where the PAHs reside, that likely causes a lower excitation of the PAHs. We have also considered the scenario where the low PAH emission is a consequence of the relative contribution of the different phases of the interstellar medium, in particular, the presence of a pervasive and highly ionised medium.

These data were useful in constraining properties (age, IMF, etc.) of the stellar content. Following the analysis previously done with the IR supernebulae C2 in NGC5253 (Martín-Hernández et al. 2005), we have constrained the stellar content of the IR compact knot in II Zw 40 using the MIR finestructure lines and strong restrictions on the nebular geometry. The same conclusions as in the case of the super-star cluster C2 were reached, i.e. we find two possible solutions for the age and upper mass cutoff of II Zw 40: 1) a young ( $\$ 3-4$ Myr) cluster with a low upper mass cutoff $M_{\text {up }}<50 M_{\odot}$; and 2) a cluster of 4-7 Myr with a standard high upper mass cutoff $M_{\text {up }} \sim 100 M_{\odot}$. We show however that the presence of multiple clusters within the compact IR, suggested by radio high spatial resolution observations, might lead to a lowering of the ionisation parameter and thus to a reconciliation between the observations and the models with a standard upper mass cutoff of $100 M_{\odot}$.

Acknowledgements. We thank the referee for his/her critical reading and highly constructive comments. NLMH thanks the "Juan de la Cierva" programme of the Spanish Ministry of Education and Science. DS wishes to thank Bernhard Brandl, Paul Crowther, Kelsey Johnson, Christophe Morisset and Bill Vacca for interesting discussions. EP acknowledges the support of the The National Research Council. Part of this work was funded by the Swiss National Science Foundation.

\section{References}

Aguero, E. L., \& Lipari, S. L. 1991, Ap\&SS, 175, 253

Allamandola, L. J., Tielens, A. G. G. M., \& Barker, J. R. 1989, ApJS, 71, 733

Allen, D. A., Wright, A. E., \& Goss, W. M. 1976, MNRAS, 177, 91

Allende Prieto, C., Lambert, D. L., \& Asplund, M. 2001, ApJ, 556, L63

Alonso-Herrero, A., Takagi, T., Baker, A. J., et al. 2004, ApJ, 612, 222

Antia, H. M., \& Basu, S. 2005, ApJ, 620, L129

Audouze, J. 1987, in Observational Cosmology, IAU Symp., 124, 89

Bahcall, J. N., Basu, S., \& M., S. A. 2005, ApJ, accepted,

[arXiv: astro-ph/0502563]

Baldwin, J. A., Spinrad, H., \& Terlevich, R. 1982, MNRAS, 198, 535

Beck, S. C., Kelly, D. M., \& Lacy, J. H. 1997, AJ, 114, 585 
Beck, S. C., Turner, J. L., \& Gorjian, V. 2001, AJ, 122, 1365

Beck, S. C., Turner, J. L., Langland-Shula, L. E., et al. 2002, AJ, 124, 2516

Beirao, P., Brandl, B. R., Devost, D., et al. 2006, ApJL, submitted

Böker, T., Storey, J. W. V., Krabbe, A., \& Lehmann, T. 1997, PASP, 109, 827

Cabanac, R. A., Vanzi, L., \& Sauvage, M. 2005, ApJ, 631, 252

Clavel, J., Schulz, B., Altieri, B., et al. 2000, A\&A, 357, 839

Cohen, M., Walker, R. G., Carter, B., et al. 1999, ApJ, 117, 1864

Conti, P. S. 1991, ApJ, 377, 115

Conti, P. S., \& Vacca, W. D. 1994, ApJ, 423, L97

Coziol, R., Doyon, R., \& Demers, S. 2001, MNRAS, 325, 1081

Davies, R. I., Sugai, H., \& Ward, M. J. 1998, MNRAS, 295, 43

de Vaucouleurs, G., \& de Vaucouleurs, A. 1961, Mem. R. Astron. Soc., 68, 69

Deeg, H., Brinks, E., Duric, N., Klein, U., \& Skillman, E. 1993, ApJ, 410, 626

Doyon, R., Joseph, R. D., \& Wright, G. S. 1994, ApJ, 421, 101

Doyon, R., Puxley, P. J., \& Joseph, R. D. 1992, ApJ, 397, 117

Draine, B. T. 1985 , ApJS, 57, 587

Draine, B. T., \& Lee, H. M. 1984, ApJ, 285, 89

Drake, J. J., \& Testa, P. 2005, Nature, 436, 525

Förster Schreiber, N. M., Genzel, R., Lutz, D., Kunze, D., \& Sternberg, A. 2001, ApJ, 552, 544

Ferland, G. J., Korista, K. T., Verner, D. A., et al. 1998, PASP, 110, 761

Forbes, D. A., \& Ward, M. J. 1993, ApJ, 416, 150

Galliano, F., Madden, S. C., Jones, A. P., Wilson, C. D., \& Bernard, J.-P. 2005, A\&A, 434, 867

Genzel, R., \& Cesarsky, C. J. 2000, ARA\&A, 38, 761

Genzel, R., Lutz, D., Sturm, E., et al. 1998, ApJ, 498, 579

Glass, I. S., \& Moorwood, A. F. M. 1985, MNRAS, 214, 429

Gorjian, V., Turner, J. L., \& Beck, S. C. 2001, ApJ, 554, L29

Graham, J. R., Wright, G. S., Meikle, W. P. S., Joseph, R. D., \& Bode, M. F. 1984, Nature, 310, 213

Guseva, N. G., Izotov, Y. I., \& Thuan, T. X. 2000, ApJ, 531, 776

Habing, H. J. 1968, Bulletin of the Astronomical Institute of the Netherlands, 19,421

Ho, P. T. P., Beck, S. C., \& Turner, J. L. 1990, ApJ, 349, 57

Hollenbach, D. J., Takahashi, T., \& Tielens, A. G. G. M. 1991, ApJ, 377, 192

Hony, S., Van Kerckhoven, C., Peeters, E., et al. 2001, A\&A, 370, 1030

Horne, K. 1986, PASP, 98, 609

Hunt, L., Bianchi, S., \& Maiolino, R. 2005, A\&A, 434, 849

Jaffe, W. J., Perola, G. C., \& Tarenghi, M. 1978, ApJ, 224, 808

Johansson, I. 1987, A\&A, 182, 179

Johnson, K. E. 2005, ed. R. Cesaroni, M. Felli, E. Churchwell, \& M. Walmsley, IAU Symp., 227, 413

Johnson, K. E., \& Kobulnicky, H. A. 2003, ApJ, 597, 923

Joy, M., \& Lester, D. F. 1988, ApJ, 331, 145

Kawara, K., Nishida, M., \& Phillips, M. M. 1989, ApJ, 337, 230

Klein, U., Weiland, H., \& Brinks, E. 1991, A\&A, 246, 323

Kobulnicky, H. A., \& Johnson, K. E. 1999, ApJ, 527, 154

Kobulnicky, H. A., Dickey, J. M., Sargent, A. I., Hogg, D. E., \& Conti, P. S. 1995, AJ, 110, 116

Kong, X., Charlot, S., Weiss, A., \& Cheng, F. Z. 2003, A\&A, 403, 877

Kotilainen, J. K., Moorwood, A. F. M., Ward, M. J., \& Forbes, D. A. 1996, A\&A, 305, 107

Kurtz, S., Churchwell, E., \& Wood, D. O. S. 1994, ApJS, 91, 659

Laurent, O., Mirabel, I. F., Charmandaris, V., et al. 2000, A\&A, 359, 887

Leitherer, C., Schaerer, D., Goldader, J. D., et al. 1999, ApJS, 123, 3

Lípari, S., Díaz, R., Taniguchi, Y., et al. 2000, AJ, 120, 645

Lípari, S. L., Díaz, R. J., Forte, J. C., et al. 2004, MNRAS, 354, L1

Lira, P., Ward, M., Zezas, A., Alonso-Herrero, A., \& Ueno, S. 2002, MNRAS, 330, 259

Lutz, D., Kunze, D., Spoon, H. W. W., \& Thornley, M. D. 1998, A\&A, 333, L75

Madden, S. C. 2000, New Astronomy Review, 44, 249

Madden, S. C., Galliano, F., Jones, A., \& Sauvage, M. 2006, A\&A, 446, 877

Martín-Hernández, N. L., van der Hulst, J. M., \& Tielens, A. G. G. M. 2003, A\&A, 407, 957
Martín-Hernández, N. L., Peeters, E., Morisset, C., et al. 2002, A\&A, 381, 606 Martín-Hernández, N. L., Schaerer, D., \& Sauvage, M. 2005, A\&A, 429, 449 Masegosa, J., Moles, M., \& Campos-Aguilar, A. 1994, ApJ, 420, 576

Mathis, J. S. 1990, ARA\&A, 28, 37

Moorwood, A. F. M. 1986, A\&A, 166, 4

Moorwood, A. F. M., \& Oliva, E. 1994, ApJ, 429, 602

Moran, E. C., Lehnert, M. D., \& Helfand, D. J. 1999, ApJ, 526, 649

Neff, S. G., Ulvestad, J. S., \& Campion, S. D. 2003, ApJ, 599, 1043

Negishi, T., Onaka, T., Chan, K.-W., \& Roellig, T. L. 2001, A\&A, 375, 566

Norris, R. P., \& Forbes, D. A. 1995, ApJ, 446, 594

Pagel, B. E. J. 1992, in The Stellar Populations of Galaxies, IAU Symp., 149, 133

Pagel, B. E. J., Simonson, E. A., Terlevich, R. J., \& Edmunds, M. G. 1992, MNRAS, 255, 325

Peeters, E., Hony, S., Van Kerckhoven, C., et al. 2002a, A\&A, 390, 1089

Peeters, E., Martín-Hernández, N. L., Damour, F., et al. 2002b, A\&A, 381, 571

Peeters, E., Allamandola, L. J., Hudgins, D. M., Hony, S., \& Tielens, A. G. G. M. 2004a, in Astrophysics of Dust, ed. A. N. Witt, G. C. Clayton, \& B. T. Draine, ASP, 309, 141

Peeters, E., Spoon, H. W. W., \& Tielens, A. G. G. M. 2004b, ApJ, 613, 986

Pérez-Montero, E., \& Díaz, A. I. 2003, MNRAS, 346, 105

Phillips, M. M., Aitken, D. K., \& Roche, P. F. 1984, MNRAS, 207, 25

Pilyugin, L. S. 2001, A\&A, 369, 594

Puget, J. L., \& Léger, A. 1989, ARA\&A, 27, 161

Raimann, D., Bica, E., Storchi-Bergmann, T., Melnick, J., \& Schmitt, H. 2000, MNRAS, 314, 295

Rieke, G. H., \& Low, F. J. 1972, ApJ, 176, L95

Rieke, G. H., \& Lebofsky, M. J. 1985, ApJ, 288, 618

Rigby, J. R., \& Rieke, G. H. 2004, ApJ, 606, 237

Rigopoulou, D., Lutz, D., Genzel, R., et al. 1996, A\&A, 315, L125

Roche, P. F., \& Aitken, D. K. 1984, MNRAS, 208, 481

Roche, P. F., Aitken, D. K., Smith, C. H., \& Ward, M. J. 1991, MNRAS, 248, 606

Rowan-Robinson, M., \& Crawford, J. 1989, MNRAS, 238, 523

Rubin, R. H., Simpson, J. P., Erickson, E. F., \& Haas, M. R. 1988, ApJ, 327, 377

Salpeter, E. E. 1955, ApJ, 121, 161

Sargent, W. L. W., \& Searle, L. 1970, ApJ, 162, L155

Sauvage, M., Thuan, T. X., \& Lagage, P. O. 1997, A\&A, 325, 98

Schaerer, D., \& Stasińska, G. 1999, A\&A, 345, L17

Sellgren, K., Tokunaga, A. T., \& Nakada, Y. 1990, ApJ, 349, 120

Siebenmorgen, R., Krügel, E., \& Spoon, H. W. W. 2004, A\&A, 414, 123

Sramek, R. A., \& Weedman, D. W. 1986, ApJ, 302, 640

Stasińska, G. 2005, A\&A, 434, 507

Storchi-Bergmann, T., Kinney, A. L., \& Challis, P. 1995, ApJS, 98, 103

Storey, P. J., \& Hummer, D. G. 1995, MNRAS, 272, 41

Thornley, M. D., Schreiber, N. M. F., Lutz, D., et al. 2000, ApJ, 539, 641

Tielens, A. G. G. M. 1993, in Dust and Chemistry in Astronomy, ed. T. J. Millar, \& D. A. Williams, 103

Tielens, A. G. G. M., Meixner, M. M., van der Werf, P. P., et al. 1993, Science, 262,86

Turner, J. L., Ho, P. T. P., \& Beck, S. C. 1998, AJ, 116, 1212

Vacca, W. D., \& Conti, P. S. 1992, ApJ, 401, 543

Vacca, W. D., Johnson, K. E., \& Conti, P. S. 2002, AJ, 123, 772

Vader, J. P., Frogel, J. A., Terndrup, D. M., \& Heisler, C. A. 1993, AJ, 106, 1743

Vanzi, L., \& Rieke, G. H. 1997, ApJ, 479, 694

Vanzi, L., Rieke, G. H., Martin, C. L., \& Shields, J. C. 1996, ApJ, 466, 150

Verma, A., Lutz, D., Sturm, E., et al. 2003, A\&A, 403, 829

Verstraete, L., Puget, J. L., Falgarone, E., et al. 1996, A\&A, 315, L337

Viegas, S. M., Contini, M., \& Contini, T. 1999, A\&A, 347, 112

Walsh, J. R., \& Roy, J. 1993, MNRAS, 262, 27

Westera, P., Cuisinier, F., Telles, E., \& Kehrig, C. 2004, A\&A, 423, 133

Wood, D. O. S., \& Churchwell, E. 1989, ApJS, 69, 831

Wynn-Williams, C. G., \& Becklin, E. E. 1986, ApJ, 308, 620

Young Owl, R. C., Meixner, M. M., Fong, D., et al. 2002, ApJ, 578, 885 